\title{
Ebeveyn Duygusal Erişilebilirliği, Romantik İlişki Kalitesi ve Sosyal Bağlılık ile İyi Oluş Arasındaki İlişside Yalnızlığın Aracılık Rolü*
}

\author{
Ahmet Çağlar ÖZDOĞAN**
}

- Geliş Tarihi: 16.07.2020 • Kabul Tarihi: 25.10.2020 • Çevrimiçi Yayın Tarihi: 03.11.2020

\section{$\ddot{\mathbf{O} z}$}

$\mathrm{Bu}$ araştırmada öğretmen adaylarında, psikolojik - öznel iyi oluş düzeyleri ve anne ve babaya duygusal erişilebilirlik, romantik ilişki kalitesi ve sosyal bağlılığın arasındaki ilişki yalnızlık aracılığıyla incelenmiştir. Araştırmanın katılımcıları Bozok Üniversitesi Eğitim Fakültesinde öğrenim gören, ilişkisi olan ve ailesinden ayrı yaşayan, aynı zamanda anne ve babası hayatta olan öğrencilerden oluşmaktadır. Araştırmaya 408'i kadın ve 259'u erkek olmak üzere toplam 667 kişi katılmıştır. Araştırmada Psikolojik İyi Oluş Ölçeği, Oxford Mutluluk Ölçeği Kısa Formu, Ucla Yalnızlık Ölçeği, Ebeveyn Duygusal Erişilebilirliği Ölçeği, Romantik İlişki Kalitesi Ölçeği ve Sosyal Bağlılık Ölçeği kullanılmıştır. Verilerin analizinde SPSS 23 ve Mplus 7.0 kullanılmıştır. Elde edilen veriler korelasyon ve path analizi ile sınanmıştır. Araştırmada anne ve babaya duygusal erişilebilirlik, romantik ilişki kalitesi ve sosyal bağlılı̆̆ı; öznel ve psikolojik iyi oluşu yalnızlık aracılığıyla yordadığı tespit edilmiştir.

Anahtar sözcükler: Psikolojik İyi Oluş, Öznel iyi oluş, Yalnızlık, Ebeveyn duygusal erişilebilirliği, Romantik ilişki kalitesi, Sosyal bağl1lık

Atıf:

Özdoğan, A.Ç. (2021). Ebeveyn duygusal erişilebilirliği, romantik ilişki kalitesi ve sosyal bağlılık ile iyi oluş arasındaki ilişkide yalnızlığın aracılık rolü. Pamukkale Üniversitesi Eğitim Fakültesi Dergisi, 52, 59-85.doi:10.9779.pauefd.770592

\footnotetext{
* Bu çalışmanın bir kısmı 21. Uluslararası Psikolojik Danışma ve Rehberlik Kongresinde sözlü bildiri olarak sunulmuştur.

** Arş.Gör. Ahmet Çağlar ÖZDOĞAN, Yozgat Bozok Üniversitesi, Eğitim Fakültesi, Rehberlik ve Psikolojik Danışma Anabilim Dal1, caglarozdogan@gmail.com, orcid number:0000-0003-1406-4155
} 


\section{Giriş}

Psikolojik araştırmaların büyük bir kısmı, zihinsel sağlık sorunlarının iyileştirilmesine odaklanmıştır. Buna karşılık, psikolojik sağlık odaklı çalışmalar yürüten araştırmacılar ise kişilerin gelişimlerine nelerin katkıda bulunduğunu anlamak için çaba gösterme eğilimi içerisindedir (Suldo ve Fefer, 2015). Psikolojik sağlığın geliştirilmesine yönelik bu yaklaşımlar, kişilerin daha çok olumlu özelliklerinin incelenmesi ve geliştirilmesine büyük önem vermektedir (Seligman ve Csikszentmihalyi, 2000). Psikolojik sağlık ile ilgili yapılan bu araştırmalar incelendiğinde ise iyilik hâli üzerine yoğunlaşıldığı dikkat çekmektedir.

Kavramsal olarak iyi oluş, en yalın haliyle iyi hissetmek ve etkili bir şekilde yaşamak olarak tanımlanmaktadır ve pozitif yaşam kalitesinin önemli bir parçası olarak görülmektedir (Huppert, 2009). İyi oluş alanyazını incelendiğinde iyi oluşun öznel ve psikolojik iyi oluş boyutlarının ön plana çıktığı görülmektedir. Öznel iyi oluş, iyi oluşun hedonik; psikolojik iyi oluş ise, ödamonik boyutunu oluşturmaktadır. Ödamonik boyut, kişisel olumlu işlevselliğe vurgu yaparken hedonik boyut daha çok olumlu duygulara vurgu yapmaktadır (Bojanowska ve Piotrowski, 2019). Bu çalışmada öğretmen adaylarında, bütüncül yanını oluşturması bakımından iyi oluşun her iki boyutu da ele alınmıştır.

Psikolojik iyi oluş yeterlilik, iyimserlik, ayrıca yaşamda anlam ve amacı yansıtan geniş bir kavramdır (Diener vd., 2010) ve psikolojik iyi oluş modelinde sosyal anlamda başkalarıyla güvenli ve olumlu ilişkiler kurmak oldukça önemlidir (Keyes, Shmotkin ve Ryff, 2002). Psikolojik iyi oluş düzeyleri yüksek olan kişilerin, kendilerine karşı olumlu bir görüşe sahip olduğu (öz-kabul), düşünce ve eylemlerinde bağımsız bir şekilde kararlılık gösterdiği (özerklik) ve karşılıklı güven (pozitif kişilerarası ilişkiler) içerisinde başkalarıyla yakın ilişki içinde oldukları görülmektedir (Grabel, 2017).

Mutluluk kavramı psikoloji biliminde öznel iyi oluş olarak tanımlanmaktadır (Diener, 2000). Öznel iyi oluş, bireylerin yaşamlarının duygusal ve bilişsel değerlendirmelerini ifade eder ve yapıda bireylerin yaşamlarından aldığı duygusal doyum oldukça önemlidir (Bhagchandani, 2017). Diener ve arkadaşları (2009), öznel iyi oluşu olumlu duygusal deneyimler içerisinde olmak olarak tanımlamaktadır. Öznel iyi oluşun yüksek düzeyi, olumlu duygulanımın daha yüksek; olumsuz duygulanımın ise daha düşük düzeyde hissedilmesi ile ilişkilendirilmiştir. Bu şekilde mutlu bireyler olumlu duyguları olumsuz duygulara göre daha sık yaşamaktadır ve yaşam kalitelerini yüksek olarak değerlendirmektedirler (Suldo ve Fefer, 2015). 
Psikolojik iyi oluş ve öznel iyi oluş açısından diğer insanlar ile olumlu ilişkiler içinde olmak önemlidir (Tooby ve Cosmides, 1992). Yaşam alanları arasında kişisel ilişkiler ve başkalarıyla yakın ilişki içinde olma sosyal ve duygusal gelişimde önemli bir etkendir (Myers, 2000) ve bu ilişkiler sosyal ilişkilerin yapısını oluşturarak iyi oluşu olumlu etkilemektedir (Goswami, 2012). Bu ilişkilerin kurulamadığı durumlarda ise karşılaşılan durum genellikle yalnızlıktır (Wang vd., 2018).

Yalnızlık, kişinin sosyal ortamda diğer insanlarla kurduğu ilişkileri yansıtan ve nesnel ve öznel anlamda kişilerin kurduğu ilişkilerin zayıflığına vurgu yapan önemli bir kavramdır (Cacioppo ve Cacioppo, 2014). Yalnızlık birçok araştırmada depresyon (Diehl, Jansen, Ishchanova ve Hilger-Kolb, 2018; Rahman, Bairagi, Kumar-Dey ve Nahar, 2017) ve intihar (Chang vd., 2017; Pervin ve Ferdowshi, 2016) gibi patolojik durumların göstergesi olarak betimlenmiştir. Dolayısıyla yalnızlık, insanın psikolojik anlamda mutluluğunu ve iyilik hâlini olumsuz etkileyebilecek yaşantılardan biri durumundadır (Duru, 2008a). Yalnızlık ve psikolojik sağlık temelli yapılan araştırmalar incelendiğinde de yalnızlığın psikolojik iyi oluş (Bhagchandani, 2017; Ishag, Asif ve Malik, 2018) ve öznel iyi oluş (HombradosMendieta, García-Martín ve Gómez-Jacinto, 2013; Tu ve Zhang, 2014) ile negatif yönlü anlamsal ilişkiye sahip olduğu görülmektedir. Ayrıca dönemsel olarak ele alındığında ise yapılan araştırmalarda yalnızlığın, ergenlik sonu ve üniversite yılları içerisindeki genç yetişkinlikte yoğun bir şekilde deneyimlendiği ve ruh sağllğı sorunlarına sebebiyet verdiği görülmektedir (Musiat vd., 2018; Seçim, Alpar ve Algür, 2014).

Yalnızlığın temelleri üzerine araştırma yapan Weiss'e göre (1973) ve yalnızlık ile ilgili yapılan araştırmalar incelendiğinde, sosyal destek kaynaklarının yalnızlık üzerindeki anlamsal etkisinin ön plana çıktığı görülmektedir. Bu kaynaklara ait ilgili alanyazın incelendiğinde; arkadaş ve aile ile duygusal temas eksikliği (Bondevik ve Skogstad, 1998; Hawkley, Browne ve Cacioppo, 2005), anne ve babaya güven ve erişilebilirlik (De Minzi, 2010), bağlanma (Akdoğan, 2017; Helm vd., 2020), sosyal destek ve sosyal bağl1lık (Li-Jane ve Shi-Kai, 2007; Duru, 2008b; Zhao, Kong ve Wang, 2013), romantik ilişki kalitesi ve ilişki doyumu (Elmien, Rozanne ve Alberta, 2016; Özdemir ve Tuncay, 2008; Pereira, Taysi, Orcan ve Fincham, 2014; Türk ve Yıldız, 2017) ile yüksek düzeyde ilişkili olduğu görülmektedir.

İnsanların sosyal destek kaynaklarının oluşumu ve şekillenmesinde bağlanma yaşantıları temel bir etkendir (Kumar ve Raj, 2016). Çünkü duyarlı bir ebeveynlik ile daha fazla merak eden, kendine güvenen ve bağımsız olabilen, güvenli bir şekilde bağlanma yaşantıları olan 
çocuklar yetiştirilebilmektedir. Bu şekilde güvenli bağlı çocuklar da kendini daha çabuk toparlayabilen ve sosyal anlamda yetkin birer yetişkin olma eğilimindedir (Hong ve Park, 2012). Yalnız bağlanma kuramı sadece bireyin davranışlarına ve/veya düşüncelerine odaklanmaktadır ve bu durum çocukların yaşamlarındaki çok çeşitli ilişkileri ve ailenin bir bütün olarak değerlendirilme potansiyelini sınırlamaktadır (Saunders, Kraus, Barone ve Biringen, 2015). Ayrıca bağlanma kuramı daha çok çocuğun stres altındaki durumlarda ebeveynlerin veya çocuğa bakım veren kişilerin çocuğa karşı yaklaşımı ile ilişkilidir ve bu da çocuğun diğer durumlardaki yapısının göz ardı edilmesine neden olur (Easterbrooks ve Biringen, 2000). Çünkü ebeveynlerin çocuklarına yönelik duygusal hassasiyeti sadece çocukların stres altında olduğu durumlarda değil, çocukların ihtiyaç hissettiği çeşitli yaşantılarda da gerekebilmektedir (Lum ve Phares, 2005). Bağlanma kuramındaki bu sınırlılıktan dolayı son dönemlerde bağlanma kuramı temelinde oluşturulan, ebeveyn ve çocuk arasındaki ilişkinin yeni bir boyutu olan ebeveyn duygusal erişilebilirliği kavramı ilgili alanyazında dikkat çekmektedir (Biringen, 2000).

Ebeveyn duygusal erişilebilirliği kavramı; çocuk ve ebeveyn bağlanma ilişkileri temelinde oluşturulmuş bir yapıdır (Biringen, 2008) ve bu yapıda her koşulda aile ve çocuk arasındaki ilişkide duygusal bağlantının kurulabilme yeteneğine vurgu yapılır. Dolayısıyla çeşitli yaşantılardaki duygusal hassasiyetin dâhil edilmesi ile birlikte ilişkilerin duygusal ve karşılıklı yapısına ve dolayısıyla bağlanma kuramına genişlik kazandırılır (Saunders, 2015). Bundan dolayı ebeveyn duygusal erişilebilirliğinin daha bütüncül etkisi ile birlikte bireylerin psikolojik sağlığı üzerinde olumlu bir etkide bulunduğu düşünülmektedir.

Ebeveyn duygusal erişilebilirliği kavramı; çocuk ve ebeveyn bağlanma ilişkileri temelinde geniş̧letilerek oluşturulmuş ikili bir yapıdır (Biringen, 2008). Bu kavram, iki kişinin sağlıklı bir duygusal bağlantıyı paylaşma yeteneğini ifade eder ve böylece ilişkilerin duygusal ve karşılıklı kalitesini açıklar. Ayrıca her durumda ebeveyn ve çocuk arasındaki duygusal yaşantıların vurgulanması ile birlikte bağlanma türündeki ilişkilere genişlik kazandırılır (Saunders vd., 2015) ve bundan dolayı ailesi ile duygusal olarak sağlıklı ilişki içerisinde olan bireylerin üzerinde olumlu bir etki oluşturduğu düşünülmektedir.

Kişiler üzerinde etkili olan sadece aile yaşantıları ve aile içerisinde yaşanan duygusal süreçler değildir. Ergenlik döneminden itibaren giderek ailesinden özerkleşmeye başlayan bireylerin sosyal ortamdaki kişilerle kuracağı ilişkiler oldukça önemli bir hâle gelmektedir (Brown ve Bakken, 2011; Mounts, 2008). Sosyal ilişkilerin önemi ergenliğin bitimi ve ilk yetişkinliğin başladığı dönem içerisinde de devam etmektedir ve yapılan çalışmalar ilk 
yetişkinler için sosyal ortamdan alınan doyumun psikolojik sağlık açısından önemli olduğunu göstermektedir (Umberson ve Karas-Montez, 2010). Kişilerin sosyal ilişki bağlamında aldığı doyuma yönelik kavramlar incelendiğinde sosyal bağlılık kavramı ön plana çıkmaktadır. Sosyal bağlılık kavramının, sosyal destek kavramından daha farklı bir yapı oluşturduğu ve psikolojik sağlık açısından da önemli bir kavram olduğu görülmektedir (Duru, 2007; Lee, Draper ve Lee, 2001).

Sosyal bağlılık, kişinin güven duygusuyla aile veya arkadaşlardan daha geniş bir sosyal bağlam içinde, sosyal aidiyet duygusunu hissetmesi anlamına gelmektedir (Lee ve Robbins, 1995). Daha farklı bir ifade ile kültürel normlar çerçevesinde sosyal olarak yakın olma, ilişki kurma veya başkalarıyla kaynakları paylaşma derecesi olarak tanımlanır (Karcher, 2011) ve eğer kişi bu durumu oluşturamıyorsa kendini toplumdan yalıtılmış hissedebilmektedir (Lee ve Robbins, 1995). Bu durum karşısında da bireyin mücadelesi ve başa çıkma stratejileri oldukça önemli olarak görülmektedir (Lee ve Robbins, 1995). Bundan dolayı son dönemlerde sosyal izolasyonu azaltmaya ve sosyal bağlantıyı artırmaya odaklanan müdahale ve araştırmalar önem kazanmaktadır (Dang, 2014). Yapılan araştırmalar düşük düzeyde sosyal bağl1lığın depresyon ve intihar düşüncesi (Arango, Opperman, Gipson ve King, 2018) gibi patolojik durumlar ile ilişkili olduğunu ortaya koymaktadır. Ayrıca yine sosyal bağlılık alanyazını incelendiğinde yalnızlık ile sosyal bağl1lık arasındaki negatif yönlü güçlü bir ilişki olduğu dikkat çekmektedir (Ang, 2016; Duru, 2008a; Duru, 2008b; Satıc1, Uysal ve Deniz, 2016).

Üniversite yılları, romantik ilişki ve yaşam hedeflerinin oluşturulmaya başlandığı (Shulman ve Connolly, 2013) ve gelişimsel olarak da özellikle romantik ilişkilerin deneyimlenmesi gereken kritik bir dönemdir (Zimmer-Gembeck ve Petherick, 2006). Duygusal yakınlığı içeren ve diğer ilişki türlerine göre daha yoğun ve özel paylaşımların yaşandığı bir ilişki türü olan romantik ilişki; tutku, bağlanma ve yakınlık ile oluşturulan bir birliktelik olarak tanımlanmaktadır (Stenberg, 1986). Üniversite öğrencileri ile yapılan araştırmalar incelendiğinde; karşı cins ile doyurucu bir ilişki içerisinde olmayan bireylerin depresyon ve yalnızlık düzeylerinin yüksek olma eğilimi içerisinde olduğu görülmektedir (Chow, Ruhl ve Buhrmester, 2015; Durmaz, 2019). Ayrıca romantik ilişki içerisinde olan ve olmayan grupların karşılaştırılarak incelendiği bir araştırmada ise romantik ilişki içerisinde olan bireylerin azalan yalnızlık ile birlikte iyi oluş ve yaşam doyumlarında anlamlı şekilde artış görüldüğü belirlenmiştir (Adamczyk, 2016).

\section{Yalnızlığın Aracı Etkisi}


Üniversite yılları gelişim kuramları açısından Erikson’un yakınlığa karşı yalıtılmışlık dönemine denk gelmektedir ve bu dönemdeki kişilerin en büyük gelişimsel görevi başkalarıyla olumlu ilişkiler kurmak ve destekleyici romantik ilişki deneyimleyebilmek ve bu ilişsileri sürdürebilmektir. Kişi bu ilişkileri kurmada güçlük çektiği durumlarda ise yaşanan durum genellikle yalnızlık ve yalıtılmışlıktır (Erikson, 1968). Aile faktörü ise bu açıdan ele alındığında genç yetişkinliğe ilk adımı atmış kişilerin gelişimsel görevi içerisinde dünyayı keşfederken geri dönülebilecek güvenli bir liman olma özelliğini içerisinde barındırmaktadır (Işsk-Terzi ve Ergüner-Tekinalp, 2013) ve dolayısıyla kişilerin sosyalleşme sürecinde destekleyici bir unsur teşkil etmektedir (Hong ve Park, 2012).

Sosyal ilişkilerin insanların yalnızlaşmasına karşı koruyucu bir etkisi vardır (Nicolaisen ve Thorsen, 2016) ve yapılan çalışmalar da yalnızlığı önleme ve azaltmanın, iyi oluş ve psikolojik sağlık için önemli olduğunu ortaya koymaktadır. (Cacioppo ve Cacioppo, 2018; Luo, Hawkley, Waite ve Cacioppo, 2012). Dolayısıyla sosyal faktörlerin yalnızlık üzerindeki negatif yönlü doğrudan etkisi, yalnızlığın da iyi oluş düzeyleri üzerindeki negatif yönlü doğrudan etkisi yalnızlığın bu ilişkideki aracı ve dolaylı etkisine işaret etmektedir. Ayrıca araştırmacı tarafından yapılan alanyazın incelemesi sonucunda bu şekilde dolaylı etkinin varlığına ilişkin bir çalışma ile karşılaşılmamış olması bu çalışmanın nedenselliğini oluşturmaktadır. Bu genel amaç çerçevesinde araştırmanın problem durumu şu şekildedir:

1. Partneri olan ve her iki ebeveyni hayatta olup ailesinden ayrı yaşayan öğretmen adaylarının psikolojik iyi oluş, öznel iyi oluş, yalnızlık, ebeveyn duygusal erişilebilirliği, sosyal bağl1lık ve romantik ilişki kalitesi düzeyleri arasında anlamlı bir ilişki var mıdır?

2. Partneri olan ve her iki ebeveyni hayatta olup ailesinden ayrı yaşayan öğretmen adaylarının ebeveyn duygusal erişilebilirliği, sosyal bağlılık ve romantik ilişki kalitesi yalnızlık düzeylerini anlamlı şekilde yordamakta mıdır?

3. Partneri olan ve her iki ebeveyni hayatta olup ailesinden ayrı yaşayan öğretmen adaylarının yalnızlık düzeyleri öznel ve psikolojik iyi oluşlarını anlamlı şekilde yordamakta midır?

4. Partneri olan ve her iki ebeveyni hayatta olup ailesinden ayrı yaşayan öğretmen adaylarının ebeveyn duygusal erişilebilirliği, sosyal bağlılık ve romantik ilişki kalitesi psikolojik iyi oluş ve öznel iyi oluş düzeylerini yalnızlık aracılığıyla yordamakta midir? 


\section{Yöntem}

\section{Araștırmanın Modeli}

Araştırma öğretmen adaylarının ebeveyn duygusal erişilebilirliği, sosyal bağlılık ve romantik ilişki kalitesi ile psikolojik iyi oluş ve öznel iyi oluş düzeyleri arasındaki ilişkinin yalnızlık aracılığıyla sınanmasına yönelik betimsel bir çalışmadır. Bu kapsamda araştırma ilişkisel araştırma modeli çerçevesinde gerçekleştirilmiştir ve ilişkisel araştırmalar iki ya da daha fazla değişkenin birbiri üzerindeki etkisini incelemeye yönelik olarak kurulan bir araştırma modelidir (Fraenkel, Wallen ve Hyun, 2012).

\section{Çalışma Grubu}

Araştırmanın katılımcıları Bozok Üniversitesi Eğitim Fakültesinde öğrenim görmekte olan öğrencilerden oluşmaktadır. Katılımcıların yaş aralığı 17-29'dur ve araştırmaya 408'i kadın ve 259 'u erkek olmak üzere toplam 667 kişi katılmıştır. Katılımcıların yaş ortalaması ise 21.03'tür ( $\mathrm{Ss}=0.71)$. Araştırma kapsamında eğitim fakültesinin farklı bölümlerinde (İlköğretim Matematik Öğretmenliği, Fen Bilgisi Öğretmenliği, Rehberlik ve Psikolojik Danışmanlık, Sınıf Öğretmenliği, Okul Öncesi Öğretmenliği ve İngilizce Öğretmenliği) öğrenim gören öğrenciler dâhil edilerek mümkün olduğunca farklılık sağlanmaya çalışılmıştır. Çalışma grubun oluşturan öğrencilerin \%28.3'ü üniversite 1. sınıf, \%25.1'i üniversite 2. sınıf, \%23.7'si üniversite 3. sınıf ve \%22.9'si ise üniversite 4. sınıfta öğrenim görmektedirler.

\section{Veri Toplama Araçları}

Araştırmada veri toplamak amacıyla Psikolojik İyi Oluş Ölçeği (Diener ve ark., 2009), Oxford Mutluluk Ölçeği Kısa Formu (Hills ve Argyle, 2002), Ucla Yalnızlık Ölçeği 3. Versiyon (Russel, 1996), Ebeveyn Duygusal Erişilebilirliği Ölçeği (Lum ve Phares, 2005) Romantik İlişki Kalitesi Ölçeği (Fletcher, Simpson ve Thomas, 2000) ve Sosyal Bağlılık Ölçeği (Lee ve Robbins, 1995) ve araştırmacı tarafından oluşturulan demografik bilgi formu kullanılmıştır.

\section{Psikolojik İyi Oluş Ölçeği}

Psikolojik İyi Oluş Ölçeği Diener ve arkadaşları (2009) tarafından geliştirilmiş olup Türkçe'ye uyarlama çalışması Telef (2013) tarafından yapılmıştır. Ölçekte toplam 8 madde bulunmaktadır ve puanlama 1 ile 7 arasında değişmektedir. Ölçekten alınan yüksek puanlar bireylerdeki psikolojik iyilik hâlinin de yüksek olduğunu göstermektedir. Ölçeğin orijinal 
formunun geçerlik çalışmasında ölçeğin tek faktörden oluştuğu ve toplam açıklanan varyansın \%53 olduğu belirlenmiştir. Ölçeğin Cronbach alfa iç tutarlık katsayısı .87 olarak bulunmuştur ve ölçekteki maddelerin faktör yükleri .61 ile .77 arasında değişkenlik göstermektedir. Uyarlama çalışmasında da ölçek tek boyutlu bir yapı göstermiş ve Cronbach alfa iç tutarlık katsayısı .80 olarak belirlenmiştir.

\section{Oxford Mutluluk Ölçeği Klsa Formu}

Oxford Mutluluk Ölçeği Hills ve Argyle (2002) tarafindan geliştirilmiş olup Türkçeye uyarlama çalışması Doğan ve Akıncı Çötok (2011) tarafından yapılmıştır. Ölçekte toplam 8 madde bulunmaktadır ve ölçek tek boyutlu bir yapı göstermektedir. Ölçekten alınan yüksek puanlar bireydeki mutluluk düzeyinin de yüksek olduğunu göstermektedir. Ölçeğin kısa formu ve 29 maddelik uzun formu arasındaki korelasyon katsayısı .91 olarak bulunmuştur. Ölçeğin uyarlama çalışmasında maddelerden biri çalışmadığı için çıkarılmış ve toplam 7 madde üzerinden devam edilmiştir. Çalışmada iç tutarlık ve test-tekrar test güvenirlik katsayıları sırasıyla .74 ve .85 olarak belirlenmiştir.

\section{Ucla Yalnızlık Ölçeği 3. Versiyon}

Ucla Yalnızlık Ölçeği 3. Versiyonu Russell (1996) tarafından kişilerin yalnızlık düzeylerini belirlemek üzerine geliştirilmiş olup Türkçeye uyarlama çalışması Durak ve Şenol-Durak (2010) tarafindan gerçekleştirilmiştir. Ölçek 4'lü Likert tipi bir ölçektir ve toplam 20 maddesi bulunmaktadır. Ölçekten alınan yüksek puanlar yalnızlık düzeyinin de yüksek olduğunu göstermektedir. Ölçeğin orijinal formundaki iç tutarlık katsayıları .89 ile .94 arasında değişmektedir. Uyarlama çalışmasında ise Cronbach alfa katsayısı 0.90 olarak bulunmuştur.

\section{Ebeveyn Duygusal Erişilebilirliği Ölçeği}

Ebeveyn Duygusal Erişilebilirliği Ölçeği Lum ve Phares (2009) tarafından geliştirilmiştir ve Türkçeye uyarlama çalışması Gökçe (2013) tarafından yapılmıştır. Ölçekte anne ve babanın ayrı ayrı duygusal erişilebilir olma durumlarını değerlendirmek üzere 15'er maddeden toplam 30 madde bulunmaktadır. Ölçeğin geliştirilme çalışmasında Cronbach Alfa iç tutarlık katsayıları klinik olmayan örneklemde anne formu için .96, baba formu için ise .97 'dir. Klinik örneklemde ise anne formu için .92 ve baba formu için ise .93 olarak bulunmuştur. Ölçeğin uyarlama çalışmasında ise Cronbach Alfa iç tutarlık katsayısı anne formu için .95 , baba formu için .97 olarak belirlenmiştir.

Sosyal Băğlılık Ölçĕgi 
Sosyal Bağlllık Ölçeği Lee ve Robins (1995) tarafından geliştirilmiş olup Türkçeye uyarlaması ise Duru (2007) tarafindan yapılmıştır. Ölçek bireylerin sosyal ortamdaki yaşantılarını değerlendirmek amacıyla geliştirilmiş̧tir ve ölçekten alınan puanların yüksek olması sosyal bağll1ık düzeyinin de yüksek olduğuna işaret etmektedir. Ölçekte toplam 8 madde bulunmaktadır ve 6'lı Likert tipine sahiptir. Ölçeğin orijinal çalışmasında iç tutarlık katsayısı $\alpha=.91$, iki hafta arayla test-tekrar test güvenirliği .96 olarak bulunurken, Türkçeye uyarlama çalışmasında iç tutarlılı̆̆ $\alpha=.90$, test-tekrar test güvenirliği .90 olarak belirlenmiştir (Duru, 2007).

\section{Algılanan Romantik İlişki Kalitesi Ölçeği}

Algılanan Romantik İlişki Kalitesi Ölçeği Fletcher ve diğerleri (2000) tarafından geliştirilmiş ve Türkçeye uyarlama çalışmaları ise Sağkal ve Özdemir (2018) tarafından gerçekleştirilmiştir. Ölçek romantik ilişkide yaşantıları değerlendirmek amacıyla geliştirilmiştir ve ölçekten alınan yüksek puanlar ilişki kalitesinin de yüksek olduğuna işaret etmektedir. Ölçek 7'li Likert tipi ve toplam 6 maddeden oluşmaktadır. Ölçeğin orijinal formunda iç tutarlık katsayısı .85 üzerinde bulunurken, ölçeğin uyarlama çalışmalarında Cronbach alfa katsayısı .86 , yapı güvenirliği .87 ve test-tekrar test güvenirliği .81 olarak hesaplanmıştır (Sağkal ve Özdemir, 2018).

\section{İşlem}

Bozok Üniversitesi Eğitim Fakültesinin çeşitli bölümlerinde öğrenim gören öğrencilere 2019-2020 Eğim-Öğretim yılı içerisinde araştırma kapsamındaki ölçme araçları uygulanmıştır. Elde edilen verilerden flörtü olan, anne ve babasından ayrı yaşayan ve her iki ebeveyni de hayatta olan öğrencilerin verileri dikkate alınmış diğer veriler araştırma kapsamına dahil edilmemiştir. Örneklemdeki seçim kriterinin uygulanması ile ilk etapta toplam 690 gözlem ile analiz sürecine geçilmiştir. Tabachnick ve Fidel'e göre (2007) $z$ puanı $\pm 3.3(p<.001)$ aralığı dışında kalan veriler tek yönlü uç değer olarak değerlendirilebilmektedir. Bundan dolayı elde edilen değişkenler için tek yönlü uç değer analizi yapılmış ve $\pm 3.29^{\prime}$ un dışında kalan 17 kişinin yanıtları da veri setinden çıkarılmıştır. Çok yönlü uç değer incelemesinde ise Mahalonobis uzaklık değeri kullanılmış ve bu değere göre belirlenen 6 gözlem daha analiz dışında tutulmuştur. Çoklu bağlantıll1lı̆ın incelenmesi için CI (koşul indeksi), VIF (varyans şişme oranları) ve tolerans değerleri incelenmiştir. Değişkenlere ait CI değerinin 30'un altında, VIF değerinin 10' dan küçük veya tolerans değerlerinin .10 veya üzerinde olması durumunda çoklu bağlantılılı̆̆ın olmadığı söylenebilir (Hair, Anderson, Tahtam ve Black, 1998). Yapılan incelemede tüm değerlerin belirlenen 
aralıklar içerisinde olduğu belirlenmiş̧tir $(\mathrm{CI}=14.84-25.48 ; \mathrm{VIF}=.06-.09)$. Sonuç olarak gerekli varsayımlardan sonra toplam 667 öğrenci ile analize devam edilmiştir.

\section{Veri Analizi}

Araştırmada incelenen değişkenler için betimsel istatistikler hesaplanmış ve ardından değişkenler arası ilişkiler Pearson Momentler Çarpımı Korelasyon Katsayısı ile belirlenmiştir. Son aşamasında ise öznel - psikolojik iyi oluş düzeyleri ve anne ve babaya duygusal erişilebilirlik, romantik ilişki kalitesi ve sosyal bağlılığın arasındaki ilişkideki yalnızlığın aracılığı path analizi ile sınanmıştır. Belirtilen analizler IBM SPSS Statistics 23.00 ve Mplus 7.0 kullanılarak gerçekleştirilmiştir.

\section{Bulgular}

\section{Betimsel İstatistikler ve Korelasyonlar}

Araştırmada bağımlı ve bağımsız değişkenler arası ilişkileri belirlemek amacıyla Pearson korelasyon katsayıları hesaplanmış ve sonuçlar Tablo 1'de sunulmuştur.

Tablo 1. Psikolojik İyi Oluş Ölçeği, Oxford Mutluluk Ölçeği, Ucla Yalnızlık Ölçeği, Ebeveyn Duygusal Erişilebilirlik Ölçeği, Romantik İlişki Kalitesi Ölçeği ve Sosyal Bă̆lılık Ölçeği Arasındaki Korelasyon Değerleri ve Betimsel İstatistikler

\begin{tabular}{|c|c|c|c|c|c|c|c|c|c|}
\hline \multirow[t]{2}{*}{ Korelasyonlar } & \multirow[b]{2}{*}{1} & \multirow[b]{2}{*}{2} & \multirow[b]{2}{*}{3} & \multirow[b]{2}{*}{4} & \multirow[b]{2}{*}{5} & \multirow[b]{2}{*}{6} & \multicolumn{3}{|c|}{ Betimsel İstatistikler } \\
\hline & & & & & & & 7 & $\bar{X}$ & $(S s)$ \\
\hline 1. PİO & - & & & & & & & 47.75 & 4.79 \\
\hline 2. ÖİO & $.63 * *$ & - & & & & & & 26.40 & 3.90 \\
\hline 3. UYÖ & $-.58 * *$ & $-.61 * *$ & - & & & & & 32.62 & 7.86 \\
\hline 4. $\mathrm{ADE}$ & $.23 * *$ & $.25^{* *}$ & $-.26 * *$ & - & & & & 81.51 & 8.05 \\
\hline 5. BDE & $.14 * *$ & $.14 * *$ & $-.23 * *$ & $.67 * *$ & - & & & 84.60 & 6.01 \\
\hline 6. RİK & $.24 * *$ & $.27 * *$ & $-.38 * *$ & .03 & .05 & - & & 31.55 & 7.68 \\
\hline 7. SB & $.34 * *$ & $.30 * *$ & $-.37 * *$ & $.22 * *$ & $.10 * *$ & $.21 * *$ & - & 22.87 & 7.38 \\
\hline
\end{tabular}

$* \mathrm{p}<.05, * * \mathrm{p}<.01$

Tablo 1'de görüldüğü üzere psikolojik iyi oluş ve yalnızlık arasında negatif yönde $(\mathrm{r}=-.58, \mathrm{p}<.01)$, yine öznel iyi oluş ile arasında negatif yönde $(\mathrm{r}=-.61, \mathrm{p}<.01)$ anlamlı korelasyonlar belirlenmiştir. Psikolojik iyi oluş ve anneye duygusal erişilebilirlik $(r=.23$, $\mathrm{p}<.01)$, babaya duygusal erişilebilirlik $(\mathrm{r}=.14, \mathrm{p}<.01)$, romantik ilişski kalitesi $(\mathrm{r}=.24, \mathrm{p}<.01)$ ve sosyal bağlılık arasında $(r=.34, \mathrm{p}<.01)$ pozitif yönde anlamlı ilişkiler belirlenmiştir. 
Öznel iyi oluş ve anneye duygusal erişilebilirlik $(\mathrm{r}=.25, \mathrm{p}<.01)$, babaya duygusal erişilebilirlik $(\mathrm{r}=.14, \mathrm{p}<.01)$, romantik ilişki kalitesi $(\mathrm{r}=.27, \mathrm{p}<.01)$ ve sosyal bağl1lık arasında $(r=.30, p<.01)$ pozitif yönde anlamlı ilişkiler belirlenmiştir. Ayrıca yalnızlık ile anneye duygusal erişilebilirlik $(r=.26, p<.01)$, babaya duygusal erişilebilirlik $(r=.23$, $\mathrm{p}<.01)$, romantik ilişki kalitesi $(\mathrm{r}=.38, \mathrm{p}<.01)$ ve sosyal bağlılık arasında $(\mathrm{r}=.37, \mathrm{p}<.01)$ negatif yönde anlamlı ilişkiler belirlenmiştir.

\section{Ölçme Modelinin Test Edilmesine İlişkin Bulgular}

Hipotetik modelin test edilmesinden önce araştırmadaki değişkenlerin ölçme modeline uygunluğu test edilmiştir. $\mathrm{Bu}$ kapsamda değişkenler doğrulayıcı faktör analizi ile sınanmıştır.

Psikolojik İyi Oluş Ölçeği'ne ilişkin yapılan doğrulayıcı faktör analizinde elde edilen modelin $\mathrm{t}$ değerlerinin 11.27 ve 22.21 arasında değişmekte olduğu ve .01 düzeyinde anlamlılık gösterdiği belirlenmiştir. Bu modele ait uyum indekslerin incelendiğinde $((\chi 2(20)$ $=83.881=4.19, \mathrm{p}<.05 ; \mathrm{CFI}=.96 ; \mathrm{TLI}=.95 ; \mathrm{RMSEA}=.08 \mathrm{SRMR}=.03)$ olarak belirlenmiştir. Bulgular Psikolojik İyi Oluş Ölçeği’nin birinci düzeyde doğrulandığını göstermektedir.

Oxford Mutluluk Ölçeği’ne ilişkin yapılan doğrulayıcı faktör analizinde elde edilen modelin $\mathrm{t}$ değerlerinin 2.60 ve 7.87 arasında değişmekte olduğu ve .01 düzeyinde anlamlılık gösterdiği belirlenmiştir. $\mathrm{Bu}$ modele ait uyum indeksleri incelendiğinde $(\chi 2(14)=$ $25.961=1.85, \mathrm{p}<.05 ; \mathrm{CFI}=.97 ; \mathrm{TLI}=.95 ; \mathrm{RMSEA}=.06 \mathrm{SRMR}=.04)$ olarak belirlenmiştir. Bulgular Oxford Mutluluk Ölçeği’nin birinci düzeyde doğrulandığını göstermektedir.

Ucla Yalnızlık Ölçeği’ne ilişkin yapılan doğrulayıcı faktör analizinde elde edilen modelin $\mathrm{t}$ değerlerinin 12.61 ve 23.79 arasında değişmekte olduğu ve .01 düzeyinde anlamlılık gösterdiği belirlenmiştir. Bu modele ait uyum indeksleri incelendiğinde $(\chi 2(152)$ $=702.997=4.62, \mathrm{p}<.05 ; \mathrm{CFI}=.92 ; \mathrm{TLI}=.90 ; \mathrm{RMSEA}=.08 \mathrm{SRMR}=.04)$ olarak belirlenmiştir. Bulgular Ucla Yalnızlık Ölçeği’nin birinci düzeyde doğrulandığını göstermektedir.

Ebeveyn Duygusal Erişilebilirliği Ölçeği’nin öncelikli olarak anneye duygusal erişilebilirlik boyutuna ilişkin yapılan doğrulayıcı faktör analizinde elde edilen modelin t değerlerinin 12.27 ve 26.90 arasında değişmekte olduğu ve .01 düzeyinde anlamlılık gösterdiği belirlenmiştir. $\mathrm{Bu}$ modele ait uyum indeksleri incelendiğinde $(\chi 2(90)=$ 
275.043=3.06, $\mathrm{p}<.05 ; \mathrm{CFI}=.96 ;$ TLI $=.95 ; \mathrm{RMSEA}=.08$ SRMR $=.03$ ) olarak belirlenmiştir. Babaya duygusal erişilebilirlik boyutuna ilişkin yapılan doğrulayıcı faktör analizinde ise elde edilen modelin t değerlerinin 10.85 ve 19.85 arasında değişmekte olduğu ve .01 düzeyinde anlamlılık gösterdiği belirlenmiştir. $\mathrm{Bu}$ modele ait uyum indeksleri incelendiğinde $(\chi 2(90)=285.769=3.18, \mathrm{p}<.05 ; \mathrm{CFI}=.94 ; \mathrm{TLI}=.93 ; \mathrm{RMSEA}=.08$ SRMR $=.04$ ) olarak belirlenmiştir. Bulgular Ebeveyn Duygusal Erişilebilirliği Ölçeği'nin birinci düzeyde doğrulandığını göstermektedir.

Sosyal Bağlılık Ölçeği'ne ilişkin yapılan doğrulayıcı faktör analizinde elde edilen modelin t değerlerinin 11.16 ve 17.75 arasında değişmekte olduğu ve .01 düzeyinde anlamlılık gösterdiği belirlenmiştir. $\mathrm{Bu}$ modele ait uyum indeksleri incelendiğinde $(\chi 2(20)=$ 50.707=2.54, $\mathrm{p}<.05 ; \mathrm{CFI}=.97 ;$ TLI $=.96 ;$ RMSEA $=.08$ SRMR $=.03$ ) olarak belirlenmiştir. Bulgular Sosyal Bağlılık Ölçeği’nin birinci düzeyde doğrulandığını göstermektedir.

Algılanan Romantik İlişki Kalitesi Ölçeği’ne ilişkin yapılan doğrulayıcı faktör analizinde elde edilen modelin $\mathrm{t}$ değerlerinin 10.65 ve 15.97 arasında değişmekte olduğu ve .01 düzeyinde anlamlılık gösterdiği belirlenmiştir. $\mathrm{Bu}$ modele ait uyum indeksleri incelendiğinde $(\chi 2(9)=20.330=2.26, \mathrm{p}<.05 ; \mathrm{CFI}=.98 ; \mathrm{TLI}=.97 ; \mathrm{RMSEA}=.08 \mathrm{SRMR}=$ .02) olarak belirlenmiştir. Bulgular Algılanan Romantik İlişki Kalitesi Ölçeği’nin birinci düzeyde doğrulandı̆̆ını göstermektedir.

\section{Hipotetik Modelin Test Edilmesine İlişkin Yol Analizi Bulguları}

Öğretmen adaylarının psikolojik - öznel iyi oluş düzeyleri ve anne ve babaya duygusal erişilebilirlik, romantik ilişki kalitesi ve sosyal bağlılığı yalnızlık aracılığıyla yordayıp yordamadığını incelemek amacıyla Mplus 7.0 ile yol analizi yapılmış ve sonuçlar şekil 1'de gösterilen yapısal model ile test edilmiştir. Modeldeki bütün değişkenler gözlenen değişkenler olarak tanımlanmış ve yol analizi bu değişkenler üzerinden gerçekleştirilmiştir. Analiz sonuçlarına göre elde edilen modelin iyi uyum gösterdiği belirlenmiştir $(\chi 2(8)=$ $36.852=4,61, \mathrm{p}<.05 ; \mathrm{CFI}=.97 ; \mathrm{TLI}=.94 ; \mathrm{RMSEA}=.07 \mathrm{SRMR}=.03)$.

Modelden elde edilen parametre tahminleri incelendiğinde; anneye duygusal erişilebilirlik $(\beta=-.12)$, babaya duygusal erişilebilirlik $(\beta=-.14)$, sosyal bağlılık $(\beta=-.33)$ ve romantik ilişki kalitesi $(\beta=-.29)$ yalnızlığı negatif yönlü anlamlı olarak yordamaktadır. Yalnızlık, psikolojik iyi oluşu ( $\beta=-.35)$ negatif yönlü anlamlı düzeyde yordamaktadır. Aynı şekilde 
yalnızlık öznel iyi oluşu ( $\beta=-.30)$ negatif yönlü anlamlı şekilde yordamaktadır. Bu dört değişken birlikteyken yalnızlığa ilişkin varyansın \%28'ini açıklamaktadır.

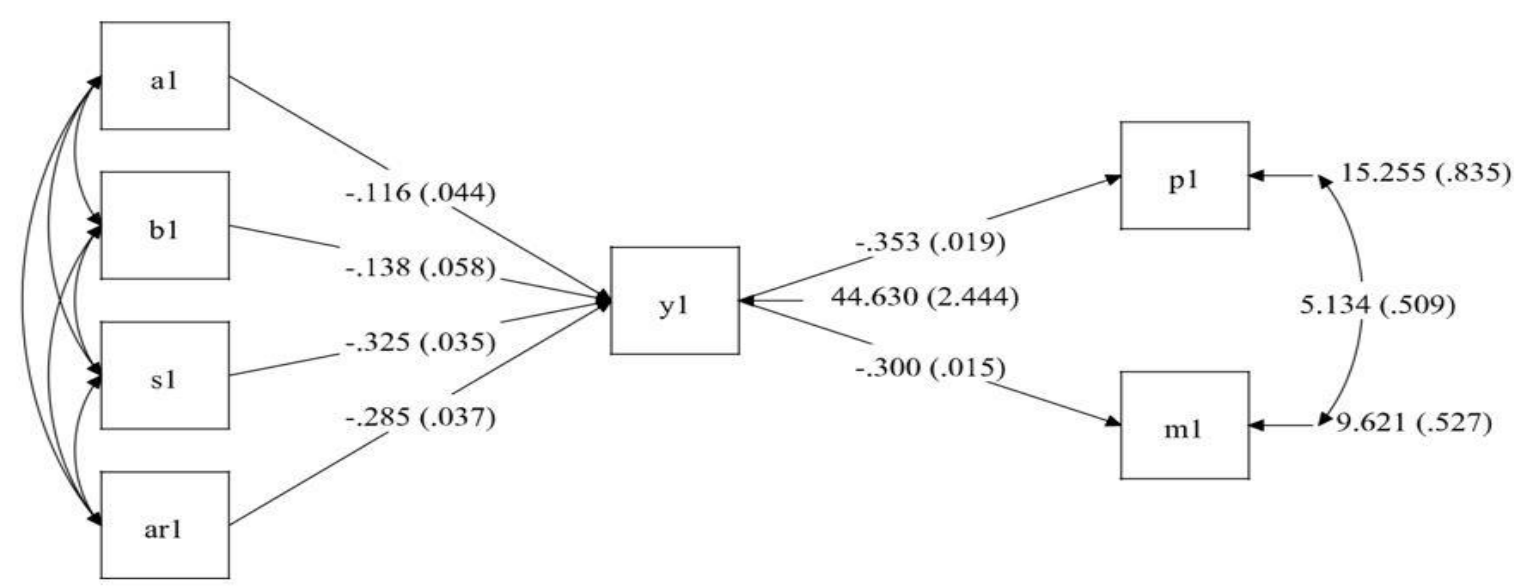

a1: Anneye Duygusal Erişilebilirlik, b1: Babaya Duygusal Erişilebilirlik, s1: Sosyal Bağlılık, ar1: Romantik İlişki Kalitesi, y1: Yalnızlık, p1: Psikolojik İyi Oluş, m1:Mutluluk

\section{Şekil I. Standartlaştırılmış Yol Katsayılarını İçeren Yol Modeli}

\section{Dolaylı Etkilere İlişkin Bulgular}

Oluşturulan modelde dolaylı etkilere ilişkin katsayılar önem sırasına göre incelendiğinde; sosyal bağlılık, psikolojik iyi oluşu $(\beta=.12)$ ve öznel iyi oluşu $(\beta=.10)$ yalnızlık aracılığıyla anlamlı şekilde yordamaktadır $(\mathrm{p}<.05)$. Romantik ilişki kalitesi psikolojik iyi oluşu $(\beta=.10)$ ve öznel iyi oluşu $(\beta=.09)$ yalnızlık aracılığıyla anlamlı şekilde yordamaktadır $(\mathrm{p}<.05)$. Babaya duygusal erişilebilirlik psikolojik iyi oluşu $(\beta=.05)$ ve öznel iyi oluşu $(\beta=.04)$ yalnızlık aracılığıyla anlamlı şekilde yordamaktadır $(\mathrm{p}<.05)$. Son olarak anneye duygusal erişilebilirlik psikolojik iyi oluşu $(\beta=.04)$ ve öznel iyi oluşu $(\beta=.04)$ yalnızlık aracılığıyla anlamlı şekilde yordamaktadır $(\mathrm{p}<.05)$. Araştırma kapsamındaki değişkenlerin yalnızlık aracılığıyla öznel ve psikolojik iyi oluş üzerindeki önem sırası sosyal bağlılık, romantik ilişki kalitesi ve anne-babaya duygusal erişilebilirlik şeklindedir. Annebabaya duygusal erişilebilirlik, sosyal bağlılık ve romantik ilişki kalitesi ile yalnızlık bir araya geldiğinde psikolojik iyi oluşa ilişkin varyansın \%34'ünü, öznel iyi oluşun ise \%37'sini açıklamaktadır. Sonuç itibariyle anne ve babaya duygusal erişilebilirlik, sosyal 
bağlılık ve romantik ilişki kalitesi arttıkça, yalnızlık azalmakta; azalan yalnızlık da psikolojik ve öznel iyi oluş düzeylerini artırmaktadır.

\section{Sonuç ve Tartışma}

$\mathrm{Bu}$ araştırmada, öğretmen adaylarında ebeveyn duygusal erişilebilirliği, sosyal bağlılık ve romantik ilişki kalitesinin psikolojik iyi oluş ve öznel iyi oluş düzeylerini yalnızlık aracılığıyla anlamlı olarak yordadığı belirlenmiştir. Elde edilen sonuçlara göre test edilen hipotetik modelin doğrulandığı görülmektedir.

\section{Modeldeki Doğrudan Etkilere İlişkin Tartışma}

$\mathrm{Bu}$ çalışmada ebeveyn duygusal erişilebilirliği, sosyal bağlılık ve romantik ilişki kalitesinin yalnızlığı; yalnızlığın da psikolojik ve öznel iyi oluşu doğrudan ve anlamlı düzeyde yordadığı tespit edilmiştir.

Araştırmada değişkenler arası yordamsal etki sırasıyla incelendiğinde ilk bulgu anne ve babaya duygusal erişilebilirliğin yalnızlığı negatif yönde anlamlı düzeyde yordamasıdır. Elde edilen bu bulguya ilişkin doğrudan yapılan bir araştırma sonucu bulunmamaktadır; fakat konunun bazı noktalarla açıklanabileceği düşünülmektedir. Ebeveyn duygusal erişilebilirliği kavramı bağlanma kuramı temelinde geliştirilen bir yapı olduğu için elde edilen sonuç bağlanma kuramı temelinde değerlendirildiğinde; bağlanma temelli yapılan araştırma sonuçları ile paralellik gösterdiği görülmektedir (Akbă̆ ve Erden-İmamoğlu, 2010; Bernadon, Babb, Hakim-Larson ve Gargg, 2011; Kıralp ve Serin, 2017; Pereira vd., 2013; Varghese ve Pistole, 2017). Ayrıca Demirli ve Demir (2014) tarafından üniversite öğrencileri üzerinde yapılan araştırmada da benzer şekilde yalnızlık ile bağlanma arasında negatif yönlü ilişki belirlenmiştir. Aynı çalışma, ailesi ile uyumu yüksek olan bireylerin yalnızlık düzeyleri üzerindeki negatif yönlü anlamsal etkiyi de ortaya koymuştur. Konu kültürel açıdan ele alındığında ise Kağıtçıbaşı'na göre (2000) karşılıklı duygusal bağlılık ve dengeli ayrışma ve bütünleşme olmak üzere ülkemizde aile ve aile niteliklerine uygun olarak iki kültürel model bulunmaktadır. Bu modellerdeki ortak nokta ise bireyselleşmenin daha çok aile bağları içerisinde ilişkisellik boyutu içerisinde oluşmasıdır ve aile içerisindeki duygusal bağımlılığın yaşamın ilerleyen dönemlerinde de devam ettirilme eğiliminde olmasıdır. Dolayısıyla ailesinden uzaklaşan üniversite öğrencilerinin aileleri ile aralarındaki duygusal bağın erişilebilir olmasının yalnızlık düzeyleri üzerinde azaltıcı bir etki oluşturduğu söylenebilir. 
Araştırmadan elde edilen bir diğer bulgu ise sosyal bağlılığın yalnızlığı negatif yönlü anlamlı bir şekilde yordamasıdır. Araştırma kapsamında elde edilen bu sonucun ilgili alanyazın ile elde edilen sonuçları desteklediği görülmektedir (Ang, 2016; Duru, 2008a; Duru, 2008b; Duru, 2008c; O'Rourke, Collins ve Sidani, 2018; Stice ve Lavner, 2018). Sosyal temas standartlarının karşılanmadığının göstergesi olan yalnızlık için tatmin edici sosyal temas yaşantıları oldukça önemlidir. Günümüzde daha çok birey yükseköğretime dâhil olmakta ve ailelerinden uzaklaşmaktadırlar ve dolayısıyla bu dönemde arkadaş ilişkileri daha da önemli hâle gelmektedir (Nicolaisen ve Thorsen, 2016). Bundan dolayı üniversite dönemi içerisinde bulunan bireylerin sosyal bağlılık düzeylerinin yalnızlık üzerindeki etkisinin beklenen bir sonuç olduğu söylenebilir.

Araştırmada öğretmen adaylarında yalnızlı̆̆ı doğrudan yordayan son bulgu ise romantik ilişki kalitesidir. Konu ile ilgili yapılan araştırmalar incelendiğinde elde edilen bulguların bu çalışmadan elde edilen bulgular ile örtüştügü görülmektedir (Chow vd., 2015; Durmaz, 2019; Dykstra ve Fokkema, 2007; Olson ve Wong, 2001; Simon, 2002; Pinquart, 2003). Ayrıca yapılan araştırmalar evli olmayan bireylerin romantik ilişki kalitelerinin psikolojik sağlıkları üzerindeki pozitif yönlü anlamsal etkisini ortaya koymaktadır (Rowsell, Coplan, 2013; Simon ve Barret, 2010). Romantik ilişki kalitesinin bireylere sağladığı sosyal desteğin, yalnızlığın olumsuz etkisini hayatlarında daha az hissedebilmelerine yol açabileceği söylenebilir. Bundan dolayı araştırma kapsamında elde edilen bulgunun ilgili alanyazından elde edilen sonuçlar ışığında beklenen bir sonuç olduğu görülmektedir.

Araştırma kapsamında elde edilen bulgular önem sırasına göre incelendiğinde; yalnızlığın en güçlü yordayıcılarının sosyal bağlılık, romantik ilişki kalitesi ve ebeveyn duygusal erişilebilirliği değişkenlerinin olduğu ve elde edilen bu bulgunun alanyazın ile tutarlılık gösterdiği görülmektedir. Duru (2008a) tarafından yalnızlı̆̆ın, sosyal destek kaynakları ve sosyal bağlılık açısından incelendiği bir araştırmada; yalnızlığın en güçlü yordayıcısının sosyal bağlılık olduğu ayrıca sosyal destek türleri açısından da yalnızlığın en güçlü yordayıcılarının sırayla arkadaş desteği, özel insan desteği ve aile desteğinin oluşturduğu görülmektedir. Bir başka araştırmada ise Kozaklı (2006), sosyal destek türleri açısından yalnızlığın en güçlü yordayıcılarının sırayla arkadaş desteği, özel insan desteği ve aile desteğinin oluşturduğunu belirlemiştir. Alanyazından elde edilen bulguların bu çalışmadan elde edilen sonuçlar ile örtüştüğü ve birbirini desteklediği görülmektedir.

Araştırmadaki doğrudan etkilere ilişkin son bulgu ise yalnızlığın psikolojik ve öznel iyi oluş düzeylerini anlamlı derecede yordamasıdır. Üniversite dönemi içerisinde bulunan 
bireylerin gelişimsel dönem itibariyle sosyalleşme düzeylerinin önemli olduğu ve bundan dolayı yalnızlık düzeylerinin iyi oluşları üzerinde belirleyici faktörlerden biri olduğu söylenebilir. Yapılan çalışmalar incelendiğinde de benzer bulguların elde edildiği görülmektedir. Bhagchandani (2017) üniversite öğrencileri üzerinde yaptığı bir çalışmada yalnızlığın psikolojik iyi oluş düzeyleri üzerindeki etkisini incelemiştir. Araştırmanın sonucuna göre yalnızlığın psikolojik iyi oluş üzerinde negatif yönde anlamlı etkisi olduğu bulunmuştur. Ayrıca Chiao, Chen ve Yi (2020) yaptıkları araştırmada yalnızlığın psikolojik iyi oluş düzeyleri üzerindeki anlamlı etkisini ortaya koymuşlardır. Psikolojik iyi oluş ve yalnızlık arasında yapılan diğer araştırmalarda da benzer sonuçların elde edildiği görülmektedir (Chen, 2019; Ishag, vd., 2018). Öznel iyi oluş ve yalnızlık arasında yapılan çalışmalar incelendiğinde de benzer şekilde yalnızlığın öznel iyi oluş üzerinde negatif düzeyde anlamlı etkisini ortaya koyan çalışmalar bulunmaktadır (Hombrados-Mendieta, García-Martín ve Gómez-Jacinto, 2013; Jin, Zhang, Wang ve An, 2020; Satıc1, 2019; Tu ve Zhang, 2014; VanderWeele vd., 2012). Farklı kültürlerden elde edilen araştırma bulguları değerlendirildiğinde sosyal ilişkilerdeki yetersizliğe işaret eden yalnızlığın, öznel ve psikolojik iyi oluş ile arasında negatif düzeyde anlamlı ilişki oluşturmasının beklenen bir sonuç olduğu söylenebilmektedir. Elde edilen bu sonucun daha önceki araştırma bulguları ile tutarlık gösterdiği ve ilgili araştırmaları desteklediği görülmektedir.

\section{Modeldeki Dolaylı Etkilere İlişkin Tartışma}

Elde edilen bulgulara göre ebeveyn duygusal erişilebilirliği, sosyal bağlılık ve romantik ilişki kalitesinin; psikolojik ve öznel iyi oluşu yalnızlık aracılığıyla yordadığı tespit edilmiştir. Daha farklı bir ifadeyle ebeveyn duygusal erişilebilirliği, sosyal bağlılık ve romantik ilişki kalitesi azaldıkça yalnızlık artmakta; artan yalnızlık da psikolojik ve öznel iyi oluş düzeylerinde azaltıcı bir etki oluşturmaktadır. İlgili alanyazında araştırmacı tarafından yapılan incelemede araştırmadaki değişkenlerle yapılan dolaylı etkilere yönelik direkt bir çalışma ile karşılaşılmamıştır; fakat çalışmanın konu olarak benzer çalışmalarla açıklanabileceği düşünülmektedir. Berkman, Glass, Brissette ve Seeman (2000) tarafindan yapılan bir araştırma aile, arkadaş ve partner tarafından algılanan sosyal desteğin yalnızlık ile dolaylı olarak iyi oluşu anlamlı olarak etkilediğini ortaya koymuştur. Çalışmada sosyal destek arttıkça yalnızlığın azalmakta olduğu ve azalan yalnızlığın da iyi oluş düzeyi üzerinde artışa neden olduğu ortaya konmuştur. Yalçın (2015) tarafından yapılan bir meta analiz çalışmasında ise iyi oluş ile aile, arkadaş ve partner tarafından algılanan sosyal destek ile yalnızlık ve depresyon ile iyi oluş değişkenleri arasındaki ilişkilerin etki büyüklükleri 
incelenmiştir. Elde edilen bulgulara göre iyi oluş değişkenleri açısından, sosyal destek ile pozitif yönlü, yalnızlık ve depresyon ile negatif yönlü bir ilişki saptanmıştır. Yapılan çalışmalardan elde edilen bulgular değerlendirildiğinde bu çalışmadan elde edilen anne ve babaya duygusal erişilebilirliğin, romantik ilişski kalitesinin ve sosyal bağlılığın yalnızlık üzerindeki anlamsal etkisiyle, psikolojik ve öznel iyi oluş düzeylerindeki anlamsal yansımalarının beklenen bir sonuç olduğu belirtilebilir.

Daha farklı araştırma sonuçları da ilgili yapıyı destekler niteliktedir. Fiori ve Consedine (2013), pozitif sosyal yaşantılar ile duygusal iyi oluş arasındaki ilişkide yalnızlığın aracı etkisini incelemiş ve yalnızlığın iki farklı pozitif yapı arasında negatif yönlü bir şekilde aracı etki oluşturduğunu ortaya koymuşlardır. Başka bir araştırmada Chen (2019), iletişim kaygısı ile iyi oluş üzerinde yalnızlığın aracı etkisini incelemiş ve sosyalleşme becerileri üzerinde engel teşkil eden iletişim kaygısı ile iyi oluş arasında yalnızlığın aracı etkisini bulgulamışlardır. Satıcı ve arkadaşları (2016) sosyal bağlılık ve yalnızlık arasında öznel mutluluğun aracı etkisini incelemişlerdir. Araştırmadan elde edilen sonuca göre sosyal bağlılık arttıkça öznel mutluluk da artmakta ve bu durum yalnızlık üzerinde azalmaya neden olmaktadır.

Bütün bu bulgulardan hareketle iyi oluş ile yalnızlığı ve aile desteğini işaret edebilen ebeveyn duygusal erişilebilirliği, partner desteğini işaret edebilen romantik ilişki kalitesi ve sosyal desteğin daha bütüncül yanını oluşturan sosyal bağlılığın negatif yönlü etkisinin bu yapılar arasındaki ilişkilerde aracı etkisinin beklenen bir sonuç olduğu ifade edilebilir.

\section{Öneriler}

Çalışmadan elde edilen sonuçlar ve metodoloji açısından araştırmacılara ve uygulayıcılara yönelik birtakım önerilerde bulunulabilir:

$\mathrm{Bu}$ araştırmadan elde edilen sonuçlar yalnızlığın psikolojik sağlık açısından negatif yönlü etkisini göstermektedir ve üniversite dönemi içerisinde genellikle ailesinden ilk defa uzaklaşan bireyler için sosyalleşme becerileri oldukça önemlidir. Dolayısıyla yalnızlığın azaltılmasına yönelik olarak özellikle lise dönemi içerisinde okul temelli yapılacak müdahalelerin (grupla psikolojik danışma, psikoeğitim vb.) önleme çalışmaları kapsamında işlevsel olacağı düşünülebilir. Aile içerisindeki duygusal etkileşim ve etkileşimin kalitesinin öğrencilerin iyi oluş düzeyleri üzerindeki anlamsal etkisinden dolayı yine önleme çalışmaları kapsamında ailelere yönelik bilgilendirici seminer çalışmaları faydalı olabilir. Ayrıca öğrencilerde sosyalleşme becerilerinin gelişimi için lise ve daha alt 
kademelerdeki öğrencilerin çeşitli sanatsal ve sportif faaliyetlere yönlendirilmeleri bu kapsamda katkı sağlayabilir. Bu faaliyetlerin aynı zamanda sosyal bağlılık düzeylerini artırıcı bir etki de oluşturması muhtemeldir.

Araştırmacılar açısından ise kültürel etmenler düşünüldüğünde araştırmanın farklı kültürlerde de yapılmasının işlevsel olacağı düşünülmektedir. Elde edilen bu bulguların farklı çalışmalarla tekrarlanması bu araştırmadan elde edilen sonucun geçerlik ve güvenirliğine katkı sağlayacaktır. Bu çalışmada nicel bir araştırma yöntemi kullanılmış ve yalnızlığın iki pozitif grup arasındaki aracı etkisi incelenmiştir. Bu doğrultuda sosyal bağlılık, romantik ilişki kalitesi ve ebeveyn duygusal erişilebilirliğinin yalnızlık üzerindeki anlamsal etkisi ortaya konmuştur. Ortaya çıkan bu bulgu nitel bir araştırma ile desteklenebilir. Yalnızlığın yordayıcılarına ilişkin derinlemesine bir inceleme ilgili alanyazına katkı sağlayacaktır. Ayrıca yalnızlığın etkisini azaltmaya yönelik programlarla yapılacak deneysel araştırmalar neden sonuç ilişkisinin kurulabilmesi açısından ve yordamsal araştırmaların bu konudaki sınırlılığından dolayı işlevsel olabileceği düşünülebilir. $\mathrm{Bu}$ araştırmada çalışma grubu açısından partneri ve her iki ebeveyni hayatta olan öğrenci grubu seçilmiştir. Yalnızlığın etkisini daha farklı açılardan görebilmek amacıyla daha farklı çalışma gruplarında araştırma tekrarlanabilir. Son olarak bu çalışmada yalnızlığın iyi oluş ve sosyal bağlılık, romantik ilişki kalitesi ve ebeveyn duygusal erişilebilirliği açısından aracı etkisi incelenmiştir. Daha farklı değişkenlerin (sosyal yetkinlik, tek başına olmayı tercih etme, arkadaşlık kalitesi, benlik saygısı ve aile iklimi, aile aidiyeti vb.) yalnızlı̆̆ın üzerindeki yordamsal etkisi incelenebilir.

Etik Kurul İzin Bilgisi: Bu araştırma, Yozgat Bozok Üniversitesi etik kurulunun 21/05/2020 tarihli 10 sayılı kararı ile alınan izinle yürütülmüştür.

Yazar Çıkar Çatışması Bilgisi: Çalışma kapsamında herhangi bir kişisel ve finansal çıkar çatışması bulunmamaktadır.

Yazar Katkısı: Makalenin hazırlanması ve düzeltilmesine ilişkin katkı ilgili yazar tarafindan gerçekleştirilmiştir. 


\section{Kaynakça}

Adamczyk, K. (2016). An investigation of loneliness and perceived social support among single and partnered young adults. Current Psychology: A Journal For Diverse Perspectives On Diverse Psychological Issues, 35(4), 674-689. https ://doi.org/10.1007/s1214 4-015-9337-7.

Akbağ, M. \& Erden-İmamoğlu, S.(2010). The prediction of gender andattachment styles on shame, guilt and loneliness. Kuram ve Uygulamada Ĕgitim Bilimleri, 10 (2), 669682.

Akdoğan, R. (2017). A model proposal on the relationships between loneliness, insecure attachment, and inferiority feelings. Personality and Individual Differences, Volume 111, 1,19-24.

Ang, C.S. (2016). Types of social connectedness and loneliness: The joint moderating effects of age and gender. Applied Research Quality Life, 11:1173-1187.

Arango, A., Opperman, K. J., Gipson, P. Y., \& King, C. A. (2016). Suicidal ideation and suicide attempts among youth who report bully victimization, bully perpetration and/or low social connectedness. Journal of Adolescence, 51, 19-29.

Berkman, L. F., Glass, T., Brissette, I., \& Seeman, T. E. (2000). From social integration to health: Durkheim in the new millennium. Social Science \& Medicine, 51(6), 843857. https://doi.org/10.1016/S0277-9536(00)00065-4.

Bernardon, S., Babb, K.A., Hakim-Larson, J. \& Gargg, M. (2011). Loneliness, attachment, and the perception and use of social supportin university students. Canadian Journal of Behavioural Science, 43,1,40-51.

Bhagchandani, R.K. (2017). "Effect of loneliness on the psychological well-being of college students". International Journal of Social Science and Humanity, 7,1,60-64.

Biringen, Z (2000). Emotional availability: Conceptualization and research findings. American Journal of Orthopsychiatry, 70, 104-114.

Biringen, Z. (2008). The emotional availability (ea) scales manual, 4th Edn. Boulder, CO: International center for excellence in emotional availability. (7).Emotional Availability: Theory, Research, and Intervention. Available from:https://www.researchgate.net/publication/280520657_Emotional_Availability _Theory_Research_and_Intervention [accessed Jan 12 2020]. 
Bojanowska, A. \& Piotrowski, K. (2019). Values and psychological well-being among adolescents - are some values 'healthier' than others? European Journal of Developmental Psychology, 16:4, 402-416, DOI: 10.1080/17405629.2018.1438257.

Bondevik, M., \& Skogstad, A. (1998). The oldest old, ADL, social network, and loneliness. Western Journal of Nursing Research, 20(3), 325-343.

Brown,B.B.,\& Bakken,J.P. (2011). Parenting and peer relationships: Reinvigorating research on family - peer linkages in adolescence. J.Res.Adolesc.21(1),153-165.

Cacioppo, J.T. \& Cacioppo, S.(2014). Social relationships and health: The toxic effects of perceived social isolation. Social and Personality Psychology Compass, 1;8(2):5872.

Cacioppo, J. T., \& Cacioppo, S. (2018). The growing problem of loneliness. The Lancet, 391(10119), 426. doi:10.1016/S0140-6736(18)30142-9.

Chang, E.C., Wana, L., Lib, P., Guoc, Y., Hed, J., Yingjie Y.W., et al. (2017). Loneliness and suicidal risk in young adults: Does believingin a changeable future help minimize suicidal risk amongthe lonely? The Journal of Psychology, Vol. 151, No. $5,453-463$.

Chen, Y. (2019). How does communication anxiety influence well-being? Examining the mediating roles of preference for online social interaction (POSI) and loneliness. International Journal of Communication, 13, 4795-4813.

Chiao, C., Chen, Y-H \& Yi, C.C. (2019). Loneliness in young adulthood: Its intersecting forms and its association with psychological well-being and family characteristics in NorthernTaiwan. PLoSONE, 14(5):e0217777.

Chow, C.M., Ruhl, H. \& Buhrmester, D. (2015). Romantic relationships and psychological distress among adolescents: Moderating role of friendship closeness. International Journal of Social Psychiatry, 1-10.

Dang, M.T. (2014). Social connectedness and self-esteem: Predictors of resilience in mental health among maltreated homeless youth. Issues in Mental Health Nursing, 35:3, 212-219, DOI: 10.3109/01612840.2013.860647.

De Minzi, M. C. R. (2010). Gender and cultural patterns of mothers' and fathers' attachment and links with children's self-competence, depression and loneliness in middle and late childhood. Early Child Development and Care, 180:1-2, 193-209, DOI: $10.1080 / 03004430903415056$. 
Demirli, A. \& Demir, A. (2014). The role of gender, attachment dimensions, and family environment on loneliness among turkish university students. Australian Journal of Guidance and Counseling, 2014; 24(1):62-75.

Diehl, K., Jansen, C., Ishchanova, K., \& Hilger-Kolb, J. (2018). Loneliness at universities: Determinants of emotional and social loneliness among students. International Journal of Environmental Research and Public Health, 29;15(9).

Diener, E. (2000). Subjective well-being: The science of happiness and a proposal for a national index. American Psychologist, 55(1), 34-43. https://doi.org/10.1037/0003$\underline{066 X .55 .1 .34}$

Diener, E., Wirtz, D., Biswas-Diener, R., Tov, W., Kim-Prieto, C., Choi, D.(2009). New measures of wellbeing. Social Indicators Research Series, 39. Doi: 10.1007/97890-481-2354-4 12.

Diener, E., Wirtz, D., Tov, W., Kim-Prieto, C., Choi, D., Oishi, S., \& Biswas-Diener, R. (2010). New well-being measures: Short scales to assess flourishing and positive and negative feelings. Social Indicators Research, 97, 143-156.

Doğan, T. ve Çötok, N. A. (2011). Oxford mutluluk ölçeği kısa formunun Türkçe uyarlaması: Geçerlik ve güvenirlik çalışması. Türk Psikolojik Danışma ve Rehberlik Dergisi, 4(36), 165-170.

Durak, M., \& Senol-Durak, E. (2010). Psychometric qualities of the Ucla loneliness scaleversion 3 as applied in a Turkish culture. Educational Gerontology, 36(10), 9881007. DOI: 10.1080/03601271003756628.

Durmaz, M.(2019). Üniversite ögrencilerinde internet kullanımı ile yalnızlık ve romantik ilişki doyum düzeyleri arasındaki ilişkinin incelenmesi. Yayınlanmış Yüksek Lisans Tezi, Yakın Doğu Üniversitesi, Sosyal Bilimler Enstitüsü, Klinik Psikoloji Anabilim Dalı, Lefkoşa.

Duru, E. (2007) Sosyal bağlılık ölçeğinin Türk kültürüne uyarlanması. Eğitim Araştırmaları Dergisi, 26, 85-94.

Duru, E. (2008a). Sosyal bağlılık ve sosyal desteğin yalnızlık üzerine doğrudan ve dolaylı rollerinin üniversiteye uyum süreci çerçevesinde incelenmesi. Türk Psikolojik Danışma ve Rehberlik Dergisi, 29 (3), 13-24. 
Duru, E. (2008b). Yalnızlığg yordamada sosyal destek ve sosyal bağlılığın rolü. Türk Psikoloji Dergisi, 24 (61), 15-24.

Duru, E. (2008c). Uyum zorluklarını yordamada yalnızlık sosyal destek ve sosyal bağlılık arasındaki ilişkilerin analizi. Kuram ve Uygulamada Eğitim Bilimleri, 8(3).

Dykstra, P. A., \& Fokkema, T. (2007). Social and emotional loneliness among divorced and married men and women: Comparing the deficit and cognitive perspectives. Basic and Applied Social Psychology, 29, 1-12.

Easterbrooks, M. A., \& Biringen, Z. (2000). Mapping the terrain of emotion availability and attachment. Attachment and Human Development, 2, 129-135.

Elmien, L., Rozanne, C. \& Alberta, S.J. (2016). Romantic relationships and loneliness in a group of South African postgraduate students. South African Review of Sociology, Vol. 47 Issue 4, p22-39. 18p.

Erikson, Erik H. (1968). Identity: Youth and Crisis. New York: Norton.

Fiori, K. L., \& Consedine, N. S. (2013). Positive and negative social exchanges and mental health across the transition to college: Loneliness as a mediator. Journal of Social and Personal Relationships, $\quad 30 \quad$ 920941. https://doi.org/10.1177/0265407512473863.

Fletcher, G. J. O., Simpson, J. A., \& Thomas, G. (2000). The measurement of perceived relationship quality components: A confirmatory factor analytic approach. Personality and Social Psychology Bulletin, 26(3), 340-354.

Fraenkel, J.R., Wallen, N.E., \& Hyun, H.H. (2012). How to design and evaluate research in education (Eight Edition). New York: McGraw-Hill.

Goswami, H. (2012). Social relationships and children's subjective well-being. Social Indicators Research, 107, 575-588. doi:10.1007/s11205-011-9864.

Gökçe, G. (2013). Ebeveynin duygusal erişilebilirliği ve genel psikolojik să̆llk:duygu düzenleme, kişilerarası ilişki tarzı ve sosyal desteğin rolü. Yayınlanmış Yüksek Lisans Tezi, Ankara Üniversitesi, Sosyal Bilimler Enstitüsü, Psikoloji Anabilim Dal, Ankara.

Grabel, B.F. (2017). The relationship between well-being and academic achievement. $A$ Systematic Revie, s0194948. 
Hair, J. F., Anderson R. E., Tahtam, R. L., \& Black, W. C. (1998). Multivariate data analysis. New Jersey:Pearson Education.

Hawkley, L. C., Browne, M. W., \& Cacioppo, J. T. (2005). How can I connect with thee? Let me count the ways. Psychological Science, 16(10), 798-804.

Helm, P.J., Jimenez T., Bultmann M., Lifshin, U., Greenberg, J. \& Arndt, J.(2020). Existential isolation, loneliness, and attachment in young adults. Personality and Individual Differences, Volume 159, 109890.

Hills, P., \& Argyle, M. (2002). The oxford happiness questionnaire: A compact scale for the measurement of psychological well-being. Personality and Individual Differences, 33 ,

1073-1082. http://dx.doi.org/10.1016/S0191-8869(01)00213-6.

Hombrados-Mendieta, I., García-Martín, M.A. \& Gómez-Jacinto, L. (2013). The relationship between social support, loneliness, and subjective well-being in a spanish sample from a multidimensional perspective. Social Indicators Research , Vol. 114, No. 3, 1013-1034.

Hong, J.R., \& Park, J.S. (2012). Impact of attachment, temperament and parenting on human development. Korean journal of pediatrics, 55(12):449-54.

Ishag G., Asif, M. \& Malik, N. (2015). Relationship of loneliness and psychological wellbeing among university hostels students: Moderating role of self-esteem. Journal of Research in Social Sciences - JRSS, Vol: 6 Number 2 ISSN: (E) 2306-112X (P) 2305- 6533.

Işık Terzi, Ş., \& Ergüner Tekinalp, B. (2013). Psikolojik Danışmada Güncel Kuramlar. Ankara: Pegem Yayınları.

Jin, Y., Zhang, M., Wang, Y. \& An, J.(2020). The relationship between trait mindfulness, loneliness, regulatory emotional self-efficacy and subjective well-being. Personality and Individual Differences, 154, 109650.

Kağıtçıbaşı, Ç. (2000). Kültürel Psikoloji - Kültür Bağlamında İnsan ve Aile. İstanbul: Evrim Yayınları.

Karcher, M. J. (2001). The Hemingway: Measure of adolescent connectedness-validation studies. Poster presented at the 109th American Psychological Conference. San Francisco, CA, August, 24th, 2001.

Karcher, M. J. (2011). The Hemingway: Measure of adolescent connectedness. Retrieved from http://www.adolescentconnectedness.com. 
Keyes, C. L. M., Shmotkin, D., \& Ryff, C. D. (2002). Optimizing well-being: The empirical encounter of two traditions. Journal of Personality and Social Psychology, 82(6), 1007-1022.

Kıralp, F.S,Ş. \& Serin, N.B. (2017). A study of students' loneliness levels and their attachment styles. Journal of Education and Training Studies, Vol. 5, No. 7.

Kozaklı, H. (2006). Üniversite Öğrencilerinde Yalnızlık ve Sosyal Destek Düzeyleri Arasındaki İlişkilerin Karşılaştırılması. Mersin Üniversitesi, Sosyal Bilimler Enstitüsü, Yüksek Lisans Tezi, Mersin.

Kumar, D. M. S., \& Raj, S. J. M. (2016). The impact of attachment styles on social competence of adolescent students. Social Sciences, 15, 1.

Luo, Y., Hawkley, L.C., Waite, L.J. \& Cacioppo, J.T. (2012). Loneliness, health, and mortality in old age: A national longitudinal study. Soc Sci Med, 74(6):907-914. doi:10.1016/j.socscimed.2011.11.028.

Lee, R. M., Draper, M. ve Lee, S. (2001). Social connectedness, dysfunctional interpersonal behaviors, and psychological distress: Testing a mediator model. Journal of Counseling Psychology, 48 (3), 310-318.

Lee, R. M., \& Robbins, S. B. (1995). Measuring belongingness: The Social Connectedness and the Social Assurance scales. Journal of Counseling Psychology, 42(2), 232241. https://doi.org/10.1037/0022- 0167.42.2.232

Li-Jane, C. \& Shi-Kai, C. (2007). Loneliness, social connectedness, and family income among undergraduate females and males in Taiwan. Social Behavior and Personality: An International Journal, Volume 35, Number 10, pp. 1353-1364(12).

Lum, J.J. ve Phares, V. (2005). Assessing the emotional availability of parents. Psychopathology and Behavioral Assessment, 27,11, 211-226.

Mounts, N. S. (2008). Linkages between parenting and peer relationships: A model for parental management of adolescents' peer relationships. In M. Kerr, H. Stattin, \& R. Engels (Eds.), What can parents do: New insights into the role of parents in adolescent problem behaviour (pp. 163-189). West Sussex, UK: Wiley.

Musiat, P., Potterton, R., Gordon, G., Spencer, L., Zeiler, M., Waldherr, K., et al. (2018). Web-based indicated prevention of common mental disorders in university students in four European countries - study protocol for a randomised controlled trial. Internet Interventions, 16, 35-42. https://doi.org/10.1016/j. invent.2018.02.004. 
Myers, D. G. (2000). The funds, friends, and faith of happy people. American Psychologist, 55(1), 56-67.

Nicolaisen, M. \& Thorsen, K.(2016). What are friends for? Friendships and loneliness over the lifespan-from 18 to 79 years. The International Journal of Aging and Human Development, 0(0) 1-33.

Pereira, M.G., Taysi, E., Orcan, F. \& Fincham, F. (2014). Attachment, infidelity, and loneliness in college students involved in a romantic relationship: The role of relationship satisfaction, morbidity, and prayer for partner. Contemporary Family Therapy, volume 36, pages333-350.

Pervin, M. M., \& Ferdowshi, N. (2016). Suicidal ideation in relation to depression, loneliness and hopelessness among university students. The Dhaka University Journal of Biological Sciences, 25(1), 57-64.

Pinquart, M. (2003). Loneliness in married, widowed, divorced, and never-married older adults. Journal of Social and Personal Relationships, 20, 31-53.

Rahman, A., Bairagi, A., Kumar Dey, B. \& Nahar, L. (2017). Loneliness and depression in university students. the chittagong university. Journal of Biological Science, vol-7 (1\&2), 2012, 175-189.

Rowsell, H. C., \& Coplan, R. J. (2013). Exploring links between shyness, romantic relationship quality, and well-being. Canadian Journal of Behavioural Science / Revue canadienne des sciences du comportement, 45(4), 287295. https://doi.org/10.1037/a0029853.

Russell, D. W. (1996). UCLA loneliness scale (Version 3): Reliability, validity, and factor structure. Journal of Personality Assessment, $66 \quad$ (1), 2040. https://doi.org/10.1207/s15327752jpa6601_2.

Sağkal, A. S. ve Özdemir, Y. (2018). Algılanan romantik ilişki kalitesi ölçeği’nin (ARİKÖ) Türkçe'ye uyarlanması: Geçerlik ve güvenirlik çalışması. Mehmet Akif Ersoy Üniversitesi Eğitim Fakültesi Dergisi, 46, 22-40. doi: 10.21764/maeuefd.329888.

Satıc1, S.A.(2019). Facebook addiction and subjective well-being: A study of the mediating role of shyness and loneliness. International Journal of Mental Health and Addiction, volume 17, pages41-55.

Satıc1, S.A., Uysal. D. \& Deniz, M.E.(2016). Linking social connectedness to loneliness: The mediating role of subjective happiness. Personality And Individual Differences, vol.97, pp.306-310, 2016. 
Saunders, H., Kraus, A., Barone, L., \& Biringen, Z. (2015). Emotional availability: Theory, research, and intervention. Frontiers in Psychology, 6, Article 1069.

Seçim, Ö., Alpar, Ö. \& Algür, S. (2014). Üniversite öğrencilerinde yalnızlık: Akdeniz Üniversitesinde yapılan ampirik bir araştırma. Elektronik Sosyal Bilimler Dergisi, Cilt:13 Sayı:48, ss.200-215.

Seligman, M., \& Csikszentmihalyi, M. (2000). Positive psychology: An introduction. American Psychologist, 55, 5-14.

Shulman, S., \& Connolly, J. (2013). The challenge of romantic relationships in emerging adulthood: Reconceptualization of the field. Emerging Adulthood, 1(1), 2739. https://doi.org/10.1177/2167696812467330.

Simon, R. W. (2002). Revisiting the relationships amonggender, marital status, and mental health. American Journal of Sociology,107, 1065 - 1096.

Simon, R. W., \& Barrett, A. E. (2010). Nonmarital romantic relationships and mental health in early adulthood: Does the association differ for women and men? Journal of Health and Social Behavior, 51(2), 168-182. doi:10.1177/0022146510372343.

Sternberg, R. J. (1986). A triangular theory of love. Psychological Review, 93(2), 119135. https://doi.org/10.1037/0033-295X.93.2.119.

Suldo, S.M., \& Fefer, S. (2015). Parent-child relationships and well-being. In C Proctor \& P.A Linley (Eds). Research applications and interventions for children and adolescents: A positive psychology perspective (pp.131-147). New York. Springer.

Stice, L.V. \& Lavner, J.A.(2018). Social connectedness and loneliness mediate the association between autistic traits and internalizing symptoms among young adults. Journal of Autism and Developmental Disorders, 49, 1096-1110.

Olson, K. L., \& Wong, E. H. (2001). Loneliness in marriage. Family Therapy, 28, 105-112.

O’Rourke, H.M., Collins, L. \& Sidani, S.(2018). Interventions to address social connectedness and loneliness for older adults: A scoping review. BMC Geriatrics, 18:214.

Özdemir, U., \& Tuncay, T. (2008). Correlates of loneliness among university students. Child and Adolescent Psychiatry and Mental Health, 2, Article 29. https://doi.org/10.1186/1753-2000-2-29.

Tabachnick, B. G, Fidel, L. S. (2007). Using Mulivariate Statistics. MA: Allyn\&Bacon, Inc.

Telef, B. B. (2013). Psikolojik iyi oluş ölçeği: Türkçeye uyarlama, geçerlik ve güvenirlik çalışması [The adaptation of psychological well-being into Turkish: A validity and reliability study]. Hacettepe University Journal of Education, 28(3), 374-384. 
Tooby J., Cosmides L. (1992). "The psychological foundations of culture," in The Adapted Mind: Evolutionary Psychology and the Generation of Culture, eds Barkow J. H., Cosmides L., Tooby J., editors. (Oxford: Oxford University Press; ), 19-136.

Tu, Y. \& Zhang, S. (2014). Loneliness and subjective well-being among chinese undergraduates: The mediating role of self-efficacy. Social Indicators Research, DOI 10.1007/s11205-014-0809-1.

Türk, E.G. \& Yıldız, A.D. (2017). Aşk biçemleri, ilişki doyumu ve yalnızlık: Üniversite öğrencileri üzerine bir çalışma. Türk Psikolojik Danışma ve Rehberlik Dergisi, (48):97-109.

Umberson, D., \& Karas Montez, J. (2010). Social relationships and health: A flashpoint for health policy. Journal of Health and Social Behavior, S54-S66.

Varghese, M.H \& Pistole, M.C. (2017). College student cyberbullying: Self-Esteem, depression, loneliness, and attachment. Journal of College Counseling, 20.

Wang, J., Mann, F., Lloyd-Evans, B., Ma, R. \& Johnson, S. (2018). Associations between loneliness and perceived social support and outcomes of mental health problems: A systematic review. BMC Psychiatry, 18:156.

Weiss, R. S. (1973). Loneliness: The experience of emotional and social isolation. Cambridge, MA: MIT Press.

Yalçın, İ. (2015). İyi oluş ve sosyal destek arasındaki ilişkiler: Türkiye'de yapılmış çalışmaların meta analizi [Relationships between well-being and social support: A meta analysis of studies conducted in Turkey]. Türk Psikiyatri Dergisi, 26(1), 2132.

Zhao, J., Kong, F. \& Wang, Y.(2013). The role of social support and self-esteem in the relationship between shyness and loneliness. Personality and Individual Differences, Volume 54, Issue 5, April 2013, Pages 577-581.

Zimmer-Gembeck, M. J. \& Petherick, J. (2006). Intimacy, dating goals and relationship satisfaction during adolescence and emerging adulthood: Identity formation, age and sex as moderators. International Journal of Behavioral Development, 30(2), 167-177. doi: 10.1177/0165025406063636. 


\title{
The Mediating Role of Loneliness in the Relationship Between Parental Emotional Availability, Romantic Relationship Quality, and Social Connectedness and Well-Being Ahmet Çağlar ÖZDOĞAN**
}

- Received: $16.07 .2020 \bullet$ Accepted: $25.10 .2020 \bullet$ Online First: 03.11.2020

\begin{abstract}
In this study, the relationship between psychological-subjective well-being levels, emotional availability to parents, quality of romantic relationships, and social connectedness of pre-service teachers were examined through loneliness. The study group of the research study consists of students who study at Bozok University Faculty of Education, have a romantic relationship, live separately from their families, and whose parents are alive. The total number of participants is 667 , 408 of which are females, and 259 are males. In this study, the Psychological Well-Being Scale, the short form of the Oxford Happiness Scale, the UCLA Loneliness Scale, the Parental Emotional Availability Scale, the Perceived Relationship Quality Components Inventory, and the Social Connectedness Scale were used. SPSS 23 and Mplus 7.0 were used to analyze the data. The data obtained were tested by correlation and path analyses. It was determined that emotional availability to parents, romantic relationship quality, and social connectedness predicted subjective and psychological well-being through loneliness.
\end{abstract}

Keywords: Psychological well-being, subjective well-being, loneliness, parental emotional availability, romantic relationship quality, social connectedness

\section{Cited:}

Özdoğan, A.Ç. (2021). The mediating role of loneliness in the relationship between parental emotional availability, romantic relationship quality, and social connectedness and wellbeing. Pamukkale University Journal of Education, 52, 59-85.doi:10.9779.pauefd.770592

\footnotetext{
* Part of this study was presented as a verbal presentation at the 21 st International Psychological Counseling and Guidance Congress.

${ }^{* *}$ Research Assistant, Ahmet Çağlar ÖZDOĞAN, Yozgat Bozok University, Faculty of Education, Department of Guidance and Psychological Counseling, caglarozdogan@gmail.com orcid number:0000-0003-1406-4155.
} 


\section{Introduction}

Most of the psychological research has focused on treating mental health diseases. On the other hand, researchers who carry out their studies with a positive psychological perspective tend to make an effort to understand what contributes to personal development (Suldo \& Fefer, 2015). Approaches for improving psychological health attach great importance to examining and developing positive traits in individuals (Seligman \& Csikszentmihalyi, 2000). When the literature on psychological health is reviewed, it comes out that they mostly focus on well-being.

As a construct, well-being is defined as feeling good and living effectively and is accepted to be an important aspect of positive life quality (Huppert, 2009). When the literature on well-being is reviewed, the dimensions of subjective well-being and psychological well-being come to the forefront. Subjective well-being is the hedonic dimension of well-being, while psychological well-being constitutes its eudemonic dimension. The eudemonic dimension emphasizes positive personal functionality, whereas the hedonic dimension mostly underlies positive feelings (Bojanowska \& Piotrowski, 2019). In this study, both dimensions of well-being have been addressed as a whole with a sample of teacher candidates.

Psychological well-being is a very comprehensive concept that consists of competence, optimism, meaning, and goal in life (Diener et al., 2010). It is considered very important to establish safe and positive social relationships with others for psychological well-being. (Keyes, Shmotkin, \& Ryff, 2002). It has been observed that people with high levels of psychological well-being have a positive view of themselves (self-acceptance), are independent in their thoughts and actions (autonomy), and are in close relations with others in mutual trust (positive interpersonal relationships). (Grabel, 2017).

The concept of happiness is defined as subjective well-being in psychology (Diener, 2000). Subjective well-being refers to individuals' emotional and cognitive evaluation of their lives (Bhagchandani, 2017). Diener and others (2009) define subjective well-being as having positive emotional experiences. A high level of subjective well-being is associated with having a higher level of positive emotions and a lower level of negative emotions. In this way, happy individuals experience positive emotions more often than negative emotions and conclude that their life is of high quality (Suldo \& Fefer, 2015). 
Having good relations with other people is important for psychological well-being and subjective well-being (Tooby \&Cosmides, 1992). Personal relations between living spaces and having close relations with others are crucial factors in social and emotional development (Myers, 2000). These relations affect well-being in a positive way by building the structure of social relations (Goswami, 2012). When these relations cannot be established, loneliness is what comes out most of the time (Wang et al., 2018).

Loneliness is an important concept that reflects one's relations established with others in a social environment and emphasizes the weakness of one's relations in subjective and objective terms (Cacioppo \& Cacioppo, 2014). Loneliness is depicted in many studies as a symptom of pathological states such as depression (Diehl, Jansen, Ishchanova, \& HilgerKolb, 2018; Rahman, Bairagi, Kumar-Dey, \& Nahar, 2017) and suicide (Chang et al., 2017; Pervin \& Ferdowshi, 2016). Because of that reason, loneliness is one of the life experiences that affect one's happiness and well-being in psychological terms (Duru, 2008a). Previous studies have indicated that loneliness has a negative relationship with psychological wellbeing (Bhagchandani, 2017; Ishag, Asif, \&Malik, 2018) and subjective well-being (Hombrados-Mendieta, García-Martín, \& Gómez-Jacinto, 2013; Tu \& Zhang, 2014). Moreover, the studies that focus on loneliness in different life phases show that loneliness is mostly experienced during young adulthood, comprising the end of puberty and university years, and leads to mental health diseases (Musiat et al., 2018; Seçim, Alpar, \& Algür, 2014).

According to Weiss (1973), who carried out a study on loneliness, the impact of social support resources on loneliness is clear. The existing literature indicated that loneliness associated with a lack of emotional contact with friends and family (Bondevik \& Skogstad, 1998; Hawkley, Browne, \& Cacioppo, 2005), trust in and access to parents (De Minzi, 2010), attachment (Akdoğan, 2017; Helm et al., 2020), social support and social commitment (Li-Jane \& Shi-Kai, 2007; Duru, 2008b; Zhao, Kong, \& Wang, 2013), the quality of the romantic relationship, and relationship satisfaction (Elmien, Rozanne, \& Alberta, 2016; Özdemir \& Tuncay, 2008; Pereira, Taysi, Orcan, \& Fincham, 2014; Türk \& Y1ld1z, 2017).

In the construction of social relations and social attitudes, individuals' attachment experience is a fundamental factor (Kumar \& Raj, 2016). This is because sensitive parenting helps bring up individuals who are more curious, self-confident, and independent who experience secure attachment. The children who are securely attached to their primary 
caregivers tend to be adults who can bounce back more easily and who are socially competent individuals (Hong \& Park, 2012). However, attachment theory focuses only on an individual's behaviors and thoughts; thus, it restricts the potential to consider various relations in children's lives and evaluate the family as a whole (Saunders, Kraus, Barone, \& Biringen, 2015). Moreover, attachment theory is mostly related to parents' or primary caregivers' attitudes towards them in conditions under stress, which causes researchers to ignore children's states in other conditions (Easterbrooks \& Biringen, 2000). Emotional sensitivity towards their children might be necessary not only in conditions where children are under stress but also in various other life experiences (Lum \& Phares, 2005). Because of this restriction in the attachment theory, recent studies in the literature have drawn attention to the concept of parental emotional availability, which is accepted to be a new dimension of the relationship between parents and children (Biringen, 2000).

Parental emotional availability is a structure built based on attachment relations between the child and parents (Biringen, 2008). It refers to the ability to build a healthy emotional attachment between the parents and the child no matter what the conditions are. Thus, the emotional and mutual structure of relations and the attachment theory is broadened by accounting for the emotional sensitivity found in various relations (Saunders et al., 2015). Therefore, it is claimed that parental emotional availability has a positive effect on individuals' psychological health.

The factors that affect individuals are not limited to life experiences within the family and their emotional processes. The relations that individuals establish with people in their social environment become quite important for those individuals who gain autonomy, starting from puberty (Brown \& Bakken, 2011; Mounts, 2008). Social relations maintain its importance as adolescence is completed and adulthood begins. Studies show that people's satisfaction in their early adulthood from their social environment is crucial for their psychological health (Umberson \& Karas-Montez, 2010). When the concepts about the satisfaction they get from their social relations are analyzed, the concept of social attachment comes to the forefront. It is clear that the concept of social attachment creates a different structure from social support and that it is very important for psychological health (Duru, 2007; Lee, Draper, \& Lee, 2001).

Social attachment means having a feeling of social belonging and reliance in a wider social environment than family and friends (Lee \& Robbins, 1995). In other words, social intimacy is defined as the degree of establishing relationships with others or sharing 
resources within the framework of cultural norms (Karcher, 2011). If the person cannot create this situation, she may feel excluded from society (Lee \& Robbins, 1995). Under this circumstance, one's struggle and coping strategies are accepted to be very important (Lee \& Robbins, 1995). Because of this reason, interventions and research on decreasing social isolation and increasing social connections have recently gained importance (Dang, 2014). Research has shown that a low level of social attachment is related to pathological conditions such as depression and suicide (Arango, Opperman, Gipson, \& King, 2018). Moreover, when the literature on social attachment is reviewed, it comes out that there is a strong negative relationship between loneliness and social attachment (Ang, 2016; Duru, 2008a; Duru, 2008b; Satıc1, Uysal, \& Deniz, 2016).

University years constitute a critical period when romantic relationships start, and life goals are established (Shulman \& Connolly, 2013). It is important to establish a romantic relationship during these years (Zimmer-Gembeck \& Petherick, 2006). A romantic relationship is described as a type of relationship that consists of emotional attachment, which is more intense and includes more special sharing than other types of relationships. Literature has indicated that those who cannot establish a satisfying romantic relationship based on passion, attachment, and closeness during this phase of life can experience depression (Stenberg, 1986). When the studies carried out on the issue are reviewed, those who do not have a satisfying relationship with a partner of the opposite sex tend to have a higher level of depression and loneliness (Chow, Ruhl, \& Buhrmester, 2015; Durmaz, 2019). Also, in a study in which groups with and without romantic relationships were compared, it was found that individuals in romantic relationships significantly increased in well-being and life satisfaction with decreased loneliness (Adamczyk, 2016).

\section{The Mediating Effect of Loneliness}

According to the theories of development, university years correspond to Erikson's period of intimacy versus isolation. In this period, people's most important developmental task is to establish positive relations with others, experience supportive romantic relationships, and maintain these relations. When people have difficulty establishing such relations, loneliness and isolation appear most of the time (Erikson, 1968). When the family factor is considered from this point of view, it is accepted to be a safe harbor where people who have just stepped into young adulthood can go back while exploring the world as a requisite of their developmental task (Iş1k-Terzi \& Ergüner-Tekinalp, 2013). Because of this reason, the family constitutes a supportive element in one’s socialization (Hong \& Park, 2012). 
Social relations have a protective effect on the feeling of loneliness (Nicolaisen \& Thorsen, 2016). Studies in the literature have found out that preventing and decreasing loneliness is important for well-being and psychological health (Cacioppo \& Cacioppo, 2018; Luo, Hawkley, Waite, \& Cacioppo, 2012). Because of this reason, this study aims at analyzing the direct effect of social factors on loneliness in a negative direction, the direct effect of loneliness on well-being in a negative direction, and the mediating and indirect effect of loneliness in this relation. In addition, the fact that the researcher has not come across any study regarding the existence of this indirect effect in the literature constitutes the causality of the current study. Within the framework of this general purpose, the research questions of the study are as follows:

1. Is there a significant relationship between the psychological well-being, subjective well-being, loneliness, parental emotional accessibility, social connectedness, and romantic relationship quality levels of teacher candidates who had a partner, whose parents are alive, and who live apart their families?

2. Do the levels of parental emotional availability, social connectedness, and quality of romantic relationships predict loneliness of teacher candidates who had a partner, whose parents are alive, and who live apart from their families at a statistically significant level?

3. Do the level of loneliness of teacher candidates who had a partner, whose parents are alive and who live apart from their families predict their level of subjective and objective well-being at a statistically significant level?

4. Do the levels of parental emotional availability, social connectedness, and quality of romantic relationships of teacher candidates who had a partner, whose parents are alive and who live apart from their families predict their level of psychological and subjective well-being via loneliness statistically significant level?

\section{Method}

\section{Research Design}

This study is a descriptive one that aims at analyzing the relationship between teacher candidates' level of parental emotional availability, social connectedness, and quality of romantic relationship on one side and psychological and subjective well-being on the other side through loneliness. The study has been carried out based on a correlational model that 
analyzes the effects of two or more variables on each other (Fraenkel, Wallen, \& Hyun, 2012).

\section{Sampling}

The sample of the study consisted of university students studying at Bozok University, Faculty of Education. The participants were between the ages of 17-29. 408 of them were female while there were 259 male participants, being 667 in total. The average age was $21.03(\mathrm{Ss}=0.71)$. The researcher tried to reach various participants, including university students studying at different majors within the Faculty of Education (Primary Mathematics Education, Science Education, Psychological Counseling and Guidance, Primary Education, Preschool Education, and Foreign Language Education). $28.3 \%$ of the participants were freshmen, $25.1 \%$ were sophomores, $23.7 \%$ were juniors, and $22.9 \%$ were seniors.

\section{Data Collection Tools}

The data collection tools used in this study are the Psychological Well-Being Scale (Diener et al., 2009), Oxford Happiness Questionnaire Short Form (Hills \& Argyle, 2002), UCLA Loneliness Scale Version 3 (Russel, 1996), Parental Emotional Availability Scale (Lum \& Phares, 2005), Perceived Relationship Quality Components Inventory (Fletcher, Simpson, \& Thomas, 2000) and Social Connectedness Scale (Lee \& Robbins, 1995) and the demographic information form developed by the researcher.

\section{Psychological Well-Being Scale}

Psychological Well-Being Scale was developed by Diener and others (2009) and adapted into Turkish culture by Telef (2013). The scale is composed of 8 items in total, where scoring changes between 1 and 7. A high score to be received at the scale implies a high level of well-being. The validity analysis carried out for the original form of the scale shows that the scale has one factor and the total variance explained is 53\%. Cronbach alfa internal consistency is .87 , and the factor load of the items included in the scale varies between .61 and .77. While the scale was adapted into Turkish culture, the one-factor structure was kept, and Cronbach alfa internal consistency coefficient was .80.

\section{Oxford Happiness Questionnaire Short Form}

Oxford Happiness Questionnaire was developed by Hills and Argyle (2002) and adapted into Turkish culture by Doğan and Akıncı Çötok (2011). The scale is composed of 8 items in total, and it has one factor. A high score to be received from the scale implies a high level of 
happiness. The correlation coefficient between the short form and the long-form having 29 items in total is found to be .91 . While the questionnaire was adapted into Turkish culture, one of the items did not work and so excluded from the questionnaire, leaving the Turkish form 7 items in total. The internal consistency and test-retest reliability coefficient were found to be .74 and .85 , respectively.

\section{UCLA Loneliness Scale Version 3}

UCLA Loneliness Scale Version 3 was developed by Russell (1996) in order to identify individuals' levels of loneliness, and it was adapted into Turkish culture by Durak and Şenol-Durak (2010). It is a 4-point Likert-type scale and consists of 20 items in total. A high score to be received from the scale implies a high level of loneliness. Internal consistency coefficients of the original scale vary between .89 and .94 . While it was adapted into Turkish culture, the Cronbach alpha coefficient was found to be 0.90 .

\section{Parental Emotional Availability Scale}

Parental Emotional Availability Scale was developed by Lum and Phares (2009) and adapted into Turkish culture by Gökçe (2013). There are 30 items in total in the scale, 15 of which aim to evaluate the mother's emotional availability and 15 of which aim to evaluate the father's emotional availability. While the scale was being developed, Cronbach Alfa internal consistency coefficients were. 96 for mothers' form and .97 for fathers' form with a non-clinical sample group. When it comes to a clinical sample, mothers' form was found to be .92, and fathers' form was found to be .93. In the Turkish adaptation of the scale, Cronbach Alfa internal coefficient was found to be .95 for mothers' form and .97 for fathers' form.

\section{Social Connectedness Scale}

Social Connectedness Scale was developed by Lee and Robins (1995) and adapted into Turkish culture by Duru (2007). The scale was developed to evaluate individuals' life experiences in social environments and a high score to be received from the scale implies a high level of social connectedness. It is a 6-point Likert-type scale having eight items in total. In the original form of the scale, the internal consistency coefficient was found to be $\alpha$ $=.91$; test-retest reliability with an interval of two weeks was found to be .96 . In Turkish adaptation of the scale, the internal consistency coefficient was found to be $\alpha=.90$; testretest reliability with an interval of two weeks was found to be .90 (Duru, 2007). 
Perceived Relationship Quality Components Inventory was developed by Fletcher and others (2000) and adapted into Turkish culture by Sağkal and Özdemir (2018). The inventory was developed to evaluate life experiences in a romantic relationship. A high score to be received from the scale implies a high level of quality in the relationship. It is a 7-point Likert-type scale having six items in total. The internal consistency coefficient of the original scale was found to be over .85 . While it was adapted into Turkish culture, the Cronbach alpha coefficient was found to be .86 , structure reliability was found to be .87 , and test-retest reliability was found to be .81 (Sağkal \& Özdemir, 2018).

\section{Procedure}

Within the study framework, data were gathered from the participants who were studying at Bozok University, Faculty of Education, in the academic year of 2019-2020. The data collected from teacher candidates who had a partner, had their parents alive, and were not living with their family were considered for the analysis. In contrast, the other data were excluded from the analysis. The criteria to choose the sample led 690 data to be included in the first phase analysis. According to Tabachnick and Fidel (2007), the scores that fall out of the interval of $z$ score $\pm 3.3(p<.001)$ are accepted to be one-way extreme value. Because of this reason, one-way extreme value analysis was done for the gathered data, and data gathered from 17 participants were excluded from the data set as they fell out of \pm 3.29 . In multiple-way extreme value analysis, Mahalanobis distance value was used, and six more data were excluded from the analysis. In order to analyze multicollinearity, CI (condition index), VIF (variance inflation factors), and tolerance values. It can be said that multicollinearity does not exist when CI value is below 30, VIF value is below ten, and tolerance values are equal to or over .10 (Hair, Anderson, Tahtam \& Black, 1998). The analysis showed that all the values were between the acceptable intervals $(\mathrm{CI}=14.84-25.48$; $\mathrm{VIF}=.06-.09)$. As a result, data gathered from 667 participant students were included in the analysis.

\section{Data Analysis}

Descriptive statistics were calculated for the variables, and then the correlations between the variables were identified via the Pearson Product-Moment Correlation Coefficient. At the final step, path analysis was used to analyze the mediating effect of loneliness in the relationship between subjective-psychological well-being, parental emotional availability, quality of the romantic relationship, and social connectedness. The analyses, as mentioned above, were utilized through IBM SPSS Statistics 23.00 and Mplus 7.0. 


\section{Results}

\section{Descriptive Statistics and Correlations}

Pearson correlation coefficients were calculated to identify the relations between dependent and independent variables, and the results are given in Table 1 below.

Table 1. Descriptive Statistics and Correlations Values between Psychological Well-Being Scale, Oxford Happiness Questionnaire Short Form, UCLA Loneliness Scale Version 3, Parental Emotional Availability Scale, Perceived Relationship Quality Components Inventory and Social Connectedness Scale

\begin{tabular}{|c|c|c|c|c|c|c|c|c|c|}
\hline & 1 & 2 & 3 & 4 & 5 & 6 & 7 & $\mathbf{M}$ & $(S D)$ \\
\hline 1. PWB & - & & & & & & & 47.75 & 4.79 \\
\hline 2. OHQ & $.63 * *$ & - & & & & & & 26.40 & 3.90 \\
\hline 3. ULS-3 & $-.58 * *$ & $-.61 * *$ & - & & & & & 32.62 & 7.86 \\
\hline 4. PEA & $.23^{* *}$ & $.25^{* *}$ & $-.26 * *$ & - & & & & 81.51 & 8.05 \\
\hline 5. PRQCI & $.14 * *$ & $.14 * *$ & $-.23 * *$ & $.67 * *$ & - & & & 84.60 & 6.01 \\
\hline 6. RIK & $.24 * *$ & $.27 * *$ & $-.38 * *$ & .03 & .05 & - & & 31.55 & 7.68 \\
\hline 7. SCS & $.34 * *$ & $.30 * *$ & $-.37 * *$ & $.22 * *$ & $.10 * *$ & $.21 * *$ & - & 22.87 & 7.38 \\
\hline
\end{tabular}

$* \mathrm{p}<.05, * * \mathrm{p}<.01$

As is seen in Table 1, there are statistically significant correlations between wellbeing and loneliness in the negative direction $(r=-.58, \mathrm{p}<.01)$, and between subjective wellbeing and loneliness in the negative direction $(r=-.61, p<.01)$. Psychological well-being has statistically significant relations in the positive direction with emotional availability of mother $(\mathrm{r}=.23, \mathrm{p}<.01)$, emotional availability of father $(\mathrm{r}=.14, \mathrm{p}<.01)$, quality of romantic relationship $(r=.24, p<.01)$ and social connectedness $(r=.34, p<.01)$. Subjective well-being has statistically significant relations in the positive way with emotional availability of mothers $(\mathrm{r}=.25, \mathrm{p}<.01)$, emotional availability of fathers $(\mathrm{r}=.14, \mathrm{p}<.01)$, quality of romantic relationship $(r=.27, p<.01)$ and social connectedness $(r=.30, p<.01)$. Moreover, loneliness has statistically significant relations in the negative way with emotional availability of mothers $(r=.26, p<.01)$, emotional availability of fathers $(r=.23, p<.01)$, quality of romantic relationships $(\mathrm{r}=.38, \mathrm{p}<.01)$ and social connectedness $(\mathrm{r}=.37, \mathrm{p}<.01)$. 


\section{Findings Regarding Testing the Measurement Model}

Before the hypothetic model was tested, the conformity of the variables in the study to the measurement model was tested. Within this framework, variables were tested via confirmatory factor analysis.

It was found out that $t$ values of the model received at the end of the confirmatory factor analysis carried out for the Psychological Well-Being Scale vary between 11.27 and 22.21 and is statistically significant at a level of .05. When the goodness of fit index belonging to this model was analyzed, the results were as below: $((\chi 2(20)=83.881=4.19, p$ $<.05 ; \mathrm{CFI}=.96 ; \mathrm{TLI}=.95 ; \mathrm{RMSEA}=.08 \mathrm{SRMR}=.03)$. These findings show that the Psychological Well-Being Scale is confirmed at the first level.

It was found out that $t$ values of the model received at the end of the confirmatory factor analysis carried out for the Oxford Happiness Questionnaire vary between 2.60 and 7.87 and is statistically significant at a level of .05 . When the goodness of fit index belonging to this model was analyzed, the results were as below: $(\chi 2(14)=25.961=1.85$, $\mathrm{p}<$ $.05 ; \mathrm{CFI}=.97 ; \mathrm{TLI}=.95 ; \mathrm{RMSEA}=.06 \mathrm{SRMR}=.04)$. These findings show that the Oxford Happiness Questionnaire is confirmed at the first level.

It was found out that $t$ values of the model received at the end of the confirmatory factor analysis carried out for the UCLA Loneliness Scale vary between 12.61 and 23.79 and is statistically significant at a level of .05 . When the goodness of fit index belonging to this model was analyzed, the results were as below: $(\chi 2(152)=702.997=4.62, \mathrm{p}<.05$; CFI $=$ $.92 ;$ TLI $=.90 ;$ RMSEA $=.08$ SRMR $=.04)$. These findings show that UCLA Loneliness Scale is confirmed at the first level.

It was found out that $t$ values of the model received at the end of the confirmatory factor analysis carried out for the dimension of mothers' availability as to the Parental Emotional Availability Scale vary between 12.27 and 26.90 and is statistically significant at a level of .05. When the goodness of fit index belonging to this model was analyzed, the results were as below: $(\chi 2(90)=275.043=3.06, \mathrm{p}<.05 ; \mathrm{CFI}=.96 ; \mathrm{TLI}=.95 ; \mathrm{RMSEA}=.08$ SRMR $=.03)$. When it comes to the dimension of father availability, it was found out that $t$ values of the model received at the end of the confirmatory factor analysis vary between 10.85 and 19.85 and are statistically significant at a level of .05. When the goodness of fit index belonging to this model was analyzed, the results were as below: $(\chi 2(90)=$ 
285.769=3.18, $\mathrm{p}<.05 ; \mathrm{CFI}=.94 ; \mathrm{TLI}=.93 ; \mathrm{RMSEA}=.08 \mathrm{SRMR}=.04)$. These findings show that the Parental Emotional Availability Scale is confirmed at the first level.

It was found out that $t$ values of the model received at the end of the confirmatory factor analysis carried out for the Social Connectedness Scale vary between 11.16 and 17.75 and is statistically significant at a level of .05. When the goodness of fit index belonging to this model was analyzed, the results were as below: $(\chi 2(20)=50.707=2.54, \mathrm{p}<.05$; CFI $=$ $.97 ;$ TLI $=.96 ;$ RMSEA $=.08$ SRMR $=.03$ ). These findings show that the Social Connectedness Scale is confirmed at the first level.

It was found out that $t$ values of the model received at the end of the confirmatory factor analysis carried out for the Perceived Relationship Quality Components Inventory vary between 10.65 and 15.97 and is statistically significant at a level of .05 . When the goodness of fit index belonging to this model was analyzed, the results were as below: $(\chi 2(9)=20.330=2.26, \mathrm{p}<.05 ; \mathrm{CFI}=.98 ; \mathrm{TLI}=.97 ; \mathrm{RMSEA}=.08 \mathrm{SRMR}=.02)$. These findings show that Perceived Relationship Quality Components Inventory is confirmed at the first level.

\section{Path Analysis Findings Regarding Testing the Hypothetic Model}

A path analysis was carried out via Mplus 7.0 in order to identify whether teacher candidates' level of psychological - subjective well-being predicts their level of parental emotional availability, quality of the romantic relationship and social connectedness with the mediating effect of loneliness or not, and the results were tested via the structural pattern given in Graph 1. All the variables in the model were defined as observable variables, and path analysis was carried out with these variables. According to the results of the analysis, it was found out that the model has an acceptable value of goodness of fit with the values below: $(\chi 2(8)=36.852=4,61, \mathrm{p}<.05 ; \mathrm{CFI}=.97 ; \mathrm{TLI}=.94 ; \mathrm{RMSEA}=.07 \mathrm{SRMR}=.03)$ 


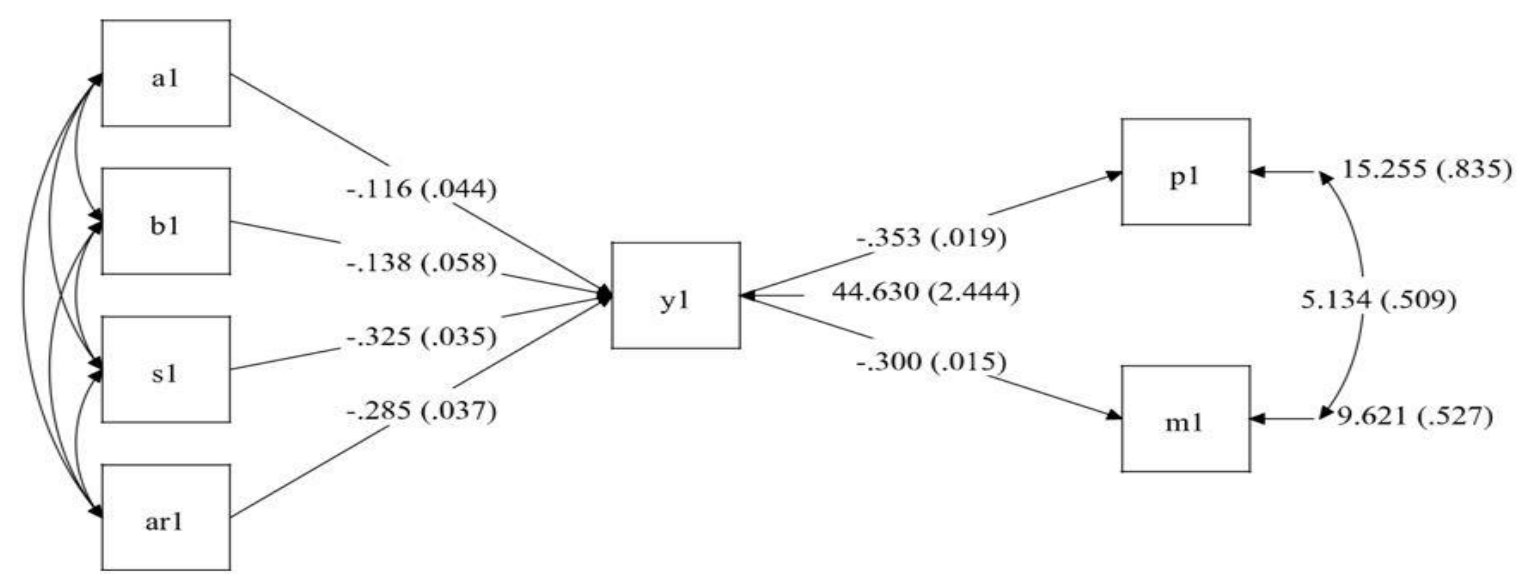

Note: a1: Emotional Availability of Mother, b1: Emotional Availability of Father, s1: Social Connectedness, ar1: Quality of Romantic Relationship, y1: Loneliness, p1: Psychological WellBeing, Happiness

Figure I. Path Model Including the Standardized Path Coefficients

When the parameter predictions gathered from the model are examined, emotional availability of mother $(\beta=-.12)$, emotional availability of father $(\beta=-.14)$, social connectedness $(\beta=-.33)$, and quality of romantic relationship $(\beta=-.29)$ predict loneliness in a negative direction. Loneliness predicts psychological well-being $(\beta=-.35)$ in a negative direction at a statistically significant level. Likewise, loneliness predicts subjective wellbeing $(\beta=-.30)$ in a negative direction at a statistically significant level. These variables together predict $28 \%$ of the variance in loneliness.

\section{Findings Regarding the Indirect Effects}

When the coefficients regarding the indirect effects within the model are examined in order of priorities, it is seen that social connectedness predicts psychological well-being $(\beta=.12)$ and subjective well-being $(\beta=.10)$ at $\mathrm{s}$ statistically significant level with the mediating effect of loneliness $(\mathrm{p}<.05)$. Quality of romantic relationship predicts psychological wellbeing $(\beta=.10)$ and subjective well-being $(\beta=.09)$ at a statistically significant level with the mediating effect of loneliness $(\mathrm{p}<.05)$. The emotional availability of the father predicts psychological well-being $(\beta=.05)$ and subjective well-being $(\beta=.04)$ at s statistically significant level with the mediating effect of loneliness $(\mathrm{p}<.05)$. Lastly, the emotional availability of the mother predicts psychological well-being $(\beta=.04)$ and subjective wellbeing $(\beta=.04)$ at a statistically significant level with the mediating effect of loneliness $(\mathrm{p}<.05)$. The study variables that are influential on subjective and psychological well-being 
with the mediating effect of loneliness are social connectedness, quality of the romantic relationship, and parental emotional availability in the order of importance. When parental emotional availability, social connectedness, and quality of romantic relationships come together with loneliness, this combination predicts $34 \%$ of the variance in psychological well-being and $37 \%$ of subjective well-being variance. As a consequence, as parental emotional availability, social connectedness, and quality of romantic relationships increase, loneliness decreases, and the diminished loneliness increases the level of psychological and subjective well-being.

\section{Discussion and Conclusion}

It has been concluded in this study that teacher candidates' level of parental emotional availability, social connectedness, and quality of romantic relationships predict their level of psychological and subjective well-being via the mediating effect of loneliness at a statistically significant level. According to the results, the hypothetic model that was tested in this study has been confirmed.

\section{Discussion on the Direct Effects in the Model}

In this study, it has been found that parental emotional availability, social connectedness, and quality of romantic relationships predict loneliness directly at a statistically significant level, where loneliness predicts psychological and subjective well-being directly at a statistically significant level.

When the predicting relations among the variables of this study are considered respectively, the first finding shows that parental emotional availability predicts loneliness in the negative direction at a statistically significant level. There is no research finding that is directly related to this result, but the issue can be explained from different viewpoints. As parental emotional availability is a concept that has been developed on the basis of attachment theory, it is deemed appropriate to evaluate this finding on the basis of attachment theory. Thus, this research finding is in parallel with other studies carried out on the basis of attachment theory (Akbağ \& Erden-İmamoğlu, 2010; Bernadon, Babb, HakimLarson, \& Gargg, 2011; Kiralp \& Serin, 2017; Pereira et al., 2013; Varghese \& Pistole, 2017). Moreover, according to a study carried out by Demirli and Demir (2014) with a study group of university students, there is a relation in the negative direction between loneliness and attachment. This study has also revealed that a high level of conformity with families has a predicting effect on loneliness in a negative direction. When the issue is considered in 
cultural terms, according to Kağıtçıbaşı (2000), two cultural models are suitable for families and family qualities in our country, which are mutual emotional attachment and balanced differentiation and integration. The common point of these models is that they put forth that individualization occurs within the family bonds on the basis of the dimension of relationality and that emotional dependency within the family tends to be kept in the following years of life. Because of this reason, it can be argued that university students' perceived level of parental emotional availability has a diminishing effect on their level of loneliness even if they go away from their families.

Another finding of this study is that social connectedness predicts loneliness negatively at a statistically significant level. This finding is in parallel with other research findings in the literature (Ang, 2016; Duru, 2008a; Duru, 2008b; Duru, 2008c; O'Rourke, Collins, \& Sidani, 2018; Stice \& Lavner, 2018). Life experiences of social contact are very important for loneliness, which indicates that social contact standards are not met. Today, more individuals receive a university education and live away from their parents, and friendship relations get more important in those years (Nicolaisen $\&$ Thorsen, 2016). Thus, it is an expected result that the level of social connectedness of individuals studying at university impacts their level of loneliness.

The last finding of this study is that romantic relationships' quality predicts teacher candidates' level of loneliness directly. It is seen that this study finding complies with other research findings in the literature (Chow et al., 2015; Durmaz, 2019; Dykstra \& Fokkema, 2007; Olson \& Wong, 2001; Simon, 2002; Pinquart, 2003). Moreover, there are studies in the literature that single individuals' quality of the romantic relationship positively affects their psychological well-being at a statistically significant level (Rowsell, Coplan, 2013; Simon \& Barret, 2010). It is thought that the social support provided by the quality of romantic relationships helps individuals feel the negative effects of loneliness to a lesser degree in their lives. Because of this reason, it can be stated that the study finding complies with the other studies that have been carried out on similar issues in the literature.

When the study findings are examined in order of importance, it is seen that the variables that predict loneliness the most are social connectedness, quality of the romantic relationship, and parental emotional and that this finding is in parallel with the findings of other studies in the literature. In a study carried out by Duru (2008a) to examine loneliness in terms of social support resources and social connectedness, it has been found out that social connectedness predicts loneliness the most, and also the other variables that most 
significantly predict loneliness support from friends, support from a special person and family support respectively. In another study conducted by Kozaklı (2006), among social support resources, the ones that predict loneliness the most are listed to support from friends, support from a special person, and family support, respectively. It is seen that the study finding complies with the findings of this study.

The final finding of the direct effects is that loneliness predicts psychological and subjective well-being statistically. It can be said that level of socialization is important for individuals who are studying at university when their developmental stage is considered, and so their level of loneliness is a determinant factor for their well-being. A literature review shows that there are similar study findings on the issue. Bhagchandani (2017) carried out a study with a study group of university students to analyze the effect of loneliness on psychological well-being. According to this study, loneliness predicts psychological wellbeing directly in a negative way. Moreover, Chiao, Chen, and Yi (2020) have found out in their study that loneliness has a statistically significant effect on psychological well-being. It is seen that other studies that focus on the relationship between loneliness and psychological well-being give similar results (Chen, 2019; Ishag et al., 2018). When studies that focus on the relation between subjective well-being and loneliness are reviewed, it is seen that loneliness has a negative effect on subjective well-being at a statistically significant level (Hombrados-Mendieta, García-Martín, \& Gómez-Jacinto, 2013; Jin, Zhang, Wang \& An, 2020; Sat1c1, 2019; Tu \& Zhang, 2014; Vander Weele et al., 2012). When studies carried out in different cultures are reviewed, it is an expected result that there is a negative relationship between loneliness, which implies an insufficiency about social relations on one side and subjective and psychological well-being on the other side. It is seen that this finding complies with other study findings in the literature.

\section{Discussion on the Indirect Effects in the Model}

According to the study's findings, parental emotional availability, social connectedness, and quality of romantic relationships predict psychological and subjective well-being with the mediating effect of loneliness. In other words, when parental emotional availability, social connectedness, and quality of romantic relationships decrease, loneliness increases, and the increased loneliness, in turn, decreases psychological and subjective well-being. As far as the literature indicates, there is no other study that focuses on direct relations between this study's variables. However, it is believed that study findings can be explained via similar studies. In a study carried out by Berkman, Glass, Brissette, and Seeman (2000), family, 
friends, and partner's social support affects loneliness and so well-being indirectly. It has been concluded in this study that as social support increases, loneliness decreases, and the decreased loneliness has an increasing effect on well-being. Another meta-analysis study conducted by Yalçın (2015) aims to analyze the extent of influence between well-being on one side and support given by family, friends, and partner on the other side, the relation between loneliness and depression on one side and well-being on the other side. According to the research findings, well-being has a positive relationship with social support and a negative relation with loneliness and depression. When various studies are considered, it is an expected result that parental emotional availability, quality of the romantic relationship, and social connectedness has a predicting effect on loneliness and on psychological and subjective well-being.

The results of different studies also support the model built in this study. Fiori and Consedine (2013) carried out a study to analyze the mediating effect of loneliness in the relationship between positive social life experiences and emotional well-being and found out that loneliness is a mediating effect in the negative direction between the two different positive structures. In another study by Chen (2019), the mediating effect of loneliness was analyzed in the relationship between communication apprehension and well-being. It was concluded that loneliness has a mediating effect on the relationship between communication apprehension and the skills of socialization and well-being. Sat1c1 and others (2016) conducted a study in which they aimed to analyze the mediating effect of subjective wellbeing in the relationship between social connectedness and loneliness. According to the study findings, as social connectedness increases, subjective well-being increases, which leads to a decrease in the level of loneliness.

Starting from all these findings, it is an expected result that well-being and loneliness, parental emotional availability which might refer to family support, quality of romantic relationship which might refer to support by the partner, and social connectedness which forms the more holistic aspect of social support have a negative relationship.

\section{Implications}

In light of the findings of the study, there are some suggestions to researchers and practitioners. The study findings point to the negative effect of loneliness on psychological health. For those in their university years and who mostly live far away from their families for the first time, socialization skills are of crucial importance. Hence, school-based preventive interventions (such as group counseling, psycho-education, etc.) to be done 
during the high-school years to decrease loneliness can be functional. As emotional interaction within the family and the quality of this interaction are important for students' well-being, seminars addressing families can be useful within preventive interventions. Moreover, directing high-school students or even younger ones to art and sports activities to help them improve their social skills. These activities are also likely to increase the level of social connectedness.

When it comes to the researchers, it would be useful to carry out a similar study in other cultures when the cultural factors are considered. Repeating similar studies will contribute to the validity and reliability of this study. This study has a qualitative research design, and the mediating effect of loneliness between two positive groups has been analyzed. In this direction, the statistically significant effect of social connectedness, quality of the romantic relationship, and parental emotional availability on loneliness have been put forth. This finding can be supported by qualitative research. A study on the variables that predict loneliness would contribute much to the related literature. Moreover, experimental studies to be conducted with programs aiming to decrease the effect of loneliness might be very functional to establish a cause and effect relationship and the restrictions of predictive studies on this issue. In this study, the study group consists of university students who have a partner and whose parents are alive. A similar study can be conducted with a different study group to see the effects of loneliness from different perspectives. Lastly, this study analyzes the mediating effect of loneliness on well-being, social connectedness, quality of the romantic relationship, and parental emotional availability. Further studies can be carried out to analyze the predictive effect of different variables (social competence, preferring to be alone, quality of friendship, self-respect, family belonging, etc.) on loneliness.

Ethical Approval: This research was carried out with the permission of Yozgat Bozok University ethics board No. 10, dated 21/05/2020.

Conflict Interest: The author declare that he has no conflict of interest.

Authors Contributions: Contribution to the preparation and revision of the article was made by the relevant author. 


\section{References}

Adamczyk, K. (2016). An investigation of loneliness and perceived social support among single and partnered young adults. Current Psychology: A Journal For Diverse Perspectives On Diverse Psychological Issues, 35(4), 674-689. https ://doi.org/10.1007/s1214 4-015-9337-7.

Akbağ, M. \& Erden-İmamoğlu, S.(2010). The prediction of gender attachment styles on shame, guilt, and loneliness. Kuram ve Uygulamada Eğitim Bilimleri, 10 (2), 669682.

Akdoğan, R. (2017). A model proposal on the relationships between loneliness, insecure attachment, and inferiority feelings. Personality and Individual Differences, Volume $111,1,19-24$.

Ang, C.S. (2016). Types of social connectedness and loneliness: The joint moderating effects of age and gender. Applied Research Quality Life, 11:1173-1187.

Arango, A., Opperman, K. J., Gipson, P. Y., \& King, C. A. (2016). Suicidal ideation and suicide attempts among youth who report bully victimization, bullying perpetration and/or low social connectedness. Journal of Adolescence, 51, 19-29.

Berkman, L. F., Glass, T., Brissette, I., \& Seeman, T. E. (2000). From social integration to health: Durkheim in the new millennium. Social Science \& Medicine, 51(6), 843857. https://doi.org/10.1016/S0277-9536(00)00065-4.

Bernardon, S., Babb, K.A., Hakim-Larson, J. \& Gargg, M. (2011). Loneliness, attachment, and the perception and use of social support in university students. Canadian Journal of Behavioural Science, 43,1,40-51.

Bhagchandani, R.K. (2017). "Effect of loneliness on the psychological well-being of college students.” International Journal of Social Science and Humanity, 7,1,60-64.

Biringen, Z (2000). Emotional availability: Conceptualization and research findings. American Journal of Orthopsychiatry, 70, 104-114.

Biringen, Z. (2008). The emotional availability (ea) scales manual, 4th Edn. Boulder, CO: International center for excellence in emotional availability. (7).Emotional Availability: Theory, Research, and Intervention. Available from:https://www.researchgate.net/publication/280520657_Emotional_Availability_ Theory_Research_and_Intervention [accessed Jan 12 2020]. 
Bojanowska, A. \& Piotrowski, K. (2019). Values and psychological well-being among adolescents - are some values 'healthier' than others? European Journal of Developmental Psychology, 16:4, 402-416, DOI: 10.1080/17405629.2018.1438257.

Bondevik, M., \& Skogstad, A. (1998). The oldest old, ADL, social network, and loneliness. Western Journal of Nursing Research, 20(3), 325-343.

Brown,B.B.,\& Bakken,J.P. (2011). Parenting and peer relationships: Reinvigorating research on family - peer linkages in adolescence. J.Res.Adolesc.21(1),153-165.

Cacioppo, J.T. \& Cacioppo, S.(2014). Social relationships and health: The toxic effects of perceived social isolation. Social and Personality Psychology Compass, 1;8(2):5872.

Cacioppo, J. T., \& Cacioppo, S. (2018). The growing problem of loneliness. The Lancet, 391(10119), 426. doi:10.1016/S0140-6736(18)30142-9.

Chang, E.C., Wana, L., Lib, P., Guoc, Y., Hed, J., Yingjie Y.W., et al. (2017). Loneliness and suicidal risk in young adults: Does believing in a changeable future help minimize suicidal risk among the lonely? The Journal of Psychology, Vol. 151, No. $5,453-463$.

Chen, Y. (2019). How does communication anxiety influence well-being? Examining the mediating roles of preference for online social interaction (POSI) and loneliness. International Journal of Communication, 13, 4795-4813.

Chiao, C., Chen, Y-H \& Yi, C.C. (2019). Loneliness in young adulthood: Its intersecting forms and its association with psychological well-being and family characteristics in NorthernTaiwan. PLoSONE, 14(5):e0217777.

Chow, C.M., Ruhl, H. \& Buhrmester, D. (2015). Romantic relationships and psychological distress among adolescents: Moderating role of friendship closeness. International Journal of Social Psychiatry, 1-10.

Dang, M.T. (2014). Social connectedness and self-esteem: Predictors of resilience in mental health among maltreated homeless youth. Issues in Mental Health Nursing, 35:3, 212-219, DOI: 10.3109/01612840.2013.860647.

De Minzi, M. C. R. (2010). Gender and cultural patterns of mothers' and fathers' attachment and links with children's self-competence, depression, and loneliness in middle and late childhood. Early Child Development and Care, 180:1-2, 193-209, DOI: $10.1080 / 03004430903415056$. 
Demirli, A. \& Demir, A. (2014). The role of gender, attachment dimensions, and family environment on loneliness among Turkish university students. Australian Journal of Guidance and Counseling, 2014; 24(1):62-75.

Diehl, K., Jansen, C., Ishchanova, K., \& Hilger-Kolb, J. (2018). Loneliness at universities: Determinants of emotional and social loneliness among students. International Journal of Environmental Research and Public Health, 29;15(9).

Diener, E. (2000). Subjective well-being: The science of happiness and a proposal for a national index. American Psychologist, 55(1), 34-43. https://doi.org/10.1037/0003$\underline{066 X .55 .1 .34}$

Diener, E., Wirtz, D., Biswas-Diener, R., Tov, W., Kim-Prieto, C., Choi, D.(2009). New measures of wellbeing. Social Indicators Research Series, 39. Doi: 10.1007/978-90481-2354-4 12.

Diener, E., Wirtz, D., Tov, W., Kim-Prieto, C., Choi, D., Oishi, S., \& Biswas-Diener, R. (2010). New well-being measures: Short scales to assess flourishing and positive and negative feelings. Social Indicators Research, 97, 143-156.

Doğan, T. ve Çötok, N. A. (2011). Oxford mutluluk ölçeği kısa formunun Türkçe uyarlaması: Geçerlik ve güvenirlik çalışması. Türk Psikolojik Danışma ve Rehberlik Dergisi, 4(36), 165-170.

Durak, M., \& Senol-Durak, E. (2010). Psychometric qualities of the UCLA loneliness scaleversion 3 as applied in Turkish culture. Educational Gerontology, 36(10), 988-1007. DOI: $10.1080 / 03601271003756628$.

Durmaz, M.(2019). Üniversite öğrencilerinde internet kullanımı ile yalnızlı ve romantik ilişki doyum düzeyleri arasındaki ilişkinin incelenmesi. Yayınlanmış Yüksek Lisans Tezi, Yakın Doğu Üniversitesi, Sosyal Bilimler Enstitüsü, Klinik Psikoloji Anabilim Dalı, Lefkoşa.

Duru, E. (2007) Sosyal bağlılık ölçeğinin Türk kültürüne uyarlanması. Eğitim Araştırmaları Dergisi, 26, 85-94.

Duru, E. (2008a). Sosyal bağlılık ve sosyal desteğin yalnızlık üzerine doğrudan ve dolaylı rollerinin üniversiteye uyum süreci çerçevesinde incelenmesi. Türk Psikolojik Danışma ve Rehberlik Dergisi, 29 (3), 13-24.

Duru, E. (2008b). Yalnızlığı yordamada sosyal destek ve sosyal bağlılığın rolü. Türk Psikoloji Dergisi, 24 (61), 15-24.

Duru, E. (2008c). Uyum zorluklarını yordamada yalnızlık sosyal destek ve sosyal bağlılık arasındaki ilişkilerin analizi. Kuram ve Uygulamada Ĕğitim Bilimleri, 8(3). 
Dykstra, P. A., \& Fokkema, T. (2007). Social and emotional loneliness among divorced and married men and women: Comparing the deficit and cognitive perspectives. Basic and Applied Social Psychology, 29, 1-12.

Easterbrooks, M. A., \& Biringen, Z. (2000). Mapping the terrain of emotion availability and attachment. Attachment and Human Development, 2, 129-135.

Elmien, L., Rozanne, C. \& Alberta, S.J. (2016). Romantic relationships and loneliness in a group of South African postgraduate students. South African Review of Sociology, 47 (4), 22-39

Erikson, Erik H. (1968). Identity: Youth and Crisis. New York: Norton.

Fiori, K. L., \& Consedine, N. S. (2013). Positive and negative social exchanges and mental health across the transition to college: Loneliness as a mediator. Journal of Social and Personal Relationships, $30 \quad$ (7), 920-941. https://doi.org/10.1177/0265407512473863.

Fletcher, G. J. O., Simpson, J. A., \& Thomas, G. (2000). The measurement of perceived relationship quality components: A confirmatory factor analytic approach. Personality and Social Psychology Bulletin, 26(3), 340-354.

Fraenkel, J.R., Wallen, N.E., \& Hyun, H.H. (2012). How to design and evaluate research in education (Eight Edition). New York: McGraw-Hill.

Goswami, H. (2012). Social relationships and children's subjective well-being. Social Indicators Research, 107, 575-588. doi:10.1007/s11205-011-9864.

Gökçe, G. (2013). Ebeveynin duygusal erişilebilirliği ve genel psikolojik sağlık:duygu düzenleme, kişilerarası ilişki tarzı ve sosyal desteğin rolü. Yayınlanmış Yüksek Lisans Tezi, Ankara Üniversitesi, Sosyal Bilimler Enstitüsü, Psikoloji Anabilim Dalı, Ankara.

Grabel, B.F. (2017). The relationship between well-being and academic achievement. $A$ Systematic Revie, s0194948.

Hair, J. F., Anderson R. E., Tahtam, R. L., \& Black, W. C. (1998). Multivariate data analysis. New Jersey: Pearson Education.

Hawkley, L. C., Browne, M. W., \& Cacioppo, J. T. (2005). How can I connect with thee? Let me count the ways. Psychological Science, 16(10), 798-804. 
Helm, P.J., Jimenez T., Bultmann M., Lifshin, U., Greenberg, J. \& Arndt, J.(2020). Existential isolation, loneliness, and attachment in young adults. Personality and Individual Differences, Volume 159, 109890.

Hills, P., \& Argyle, M. (2002). The oxford happiness questionnaire: A compact scale for the measurement of psychological well-being. Personality and Individual Differences, 33,1073-1082.http://dx.doi.org/10.1016/S0191-8869(01)00213-6.

Hombrados-Mendieta, I., García-Martín, M.A. \& Gómez-Jacinto, L. (2013). The relationship between social support, loneliness, and subjective well-being in a Spanish sample from a multidimensional perspective. Social Indicators Research, Vol. 114, No. 3, 1013-1034.

Hong, J.R., \& Park, J.S. (2012). Impact of attachment, temperament, and parenting on human development. Korean Journal of Pediatrics, 55(12):449-54.

Ishag G., Asif, M. \& Malik, N. (2015). Relationship of loneliness and psychological wellbeing among university hostels students: Moderating role of self-esteem. Journal of Research in Social Sciences - JRSS, Vol: 6 Number 2 ISSN: (E) 2306-112X (P) 2305- 6533.

Iş1k Terzi, Ş., \& Ergüner Tekinalp, B. (2013). Psikolojik Danışmada Güncel Kuramlar. Ankara: Pegem Yayınları.

Jin, Y., Zhang, M., Wang, Y. \& An, J.(2020). The relationship between trait mindfulness, loneliness, regulatory emotional self-efficacy, and subjective well-being. Personality and Individual Differences, 154, 109650.

Kağıtçıbaşı, Ç. (2000). Kültürel Psikoloji - Kültür Bă̆lamında Insan ve Aile. İstanbul: Evrim Yayınları.

Karcher, M. J. (2001). The Hemingway: Measure of adolescent connectedness-validation studies. Poster presented at the 109th American Psychological Conference. San Francisco, CA, August 24th, 2001.

Karcher, M. J. (2011). The Hemingway: Measure of adolescent connectedness. Retrieved from http://www.adolescentconnectedness.com.

Keyes, C. L. M., Shmotkin, D., \& Ryff, C. D. (2002). Optimizing well-being: The empirical encounter of two traditions. Journal of Personality and Social Psychology, 82(6), $1007-1022$.

Kıralp, F.S,Ş. \& Serin, N.B. (2017). A study of students' loneliness levels and their attachment styles. Journal of Education and Training Studies, Vol. 5, No. 7. 
Kozaklı, H. (2006). Üniversite Öğrencilerinde Yalnızlı ve Sosyal Destek Düzeyleri Arasındaki İlişkilerin Karşılaştırılması. Mersin Üniversitesi, Sosyal Bilimler Enstitüsü, Yüksek Lisans Tezi, Mersin.

Kumar, D. M. S., \& Raj, S. J. M. (2016). The impact of attachment styles on the social competence of adolescent students. Social Sciences, 15, 1.

Luo, Y., Hawkley, L.C., Waite, L.J. \& Cacioppo, J.T. (2012). Loneliness, health, and mortality in old age: A national longitudinal study. Soc Sci Med, 74(6):907-914. doi:10.1016/j.socscimed.2011.11.028.

Lee, R. M., Draper, M. ve Lee, S. (2001). Social connectedness, dysfunctional interpersonal behaviors, and psychological distress: Testing a mediator model. Journal of Counseling Psychology, 48 (3), 310-318.

Lee, R. M., \& Robbins, S. B. (1995). Measuring belongingness: The Social Connectedness and the Social Assurance scales. Journal of Counseling Psychology, 42(2), 232-241. https://doi.org/10.1037/0022- 0167.42.2.232

Li-Jane, C. \& Shi-Kai, C. (2007). Loneliness, social connectedness, and family income among undergraduate females and males in Taiwan. Social Behavior and Personality: An International Journal, Volume 35, Number 10, pp. 1353-1364(12).

Lum, J.J. ve Phares, V. (2005). Assessing the emotional availability of parents. Psychopathology and Behavioral Assessment, 27,11, 211-226.

Mounts, N. S. (2008). Linkages between parenting and peer relationships: A model for parental management of adolescents' peer relationships. In M. Kerr, H. Stattin, \& R. Engels (Eds.), What can parents do: New insights into the role of parents in adolescent problem behavior (pp. 163-189). West Sussex, UK: Wiley.

Musiat, P., Potterton, R., Gordon, G., Spencer, L., Zeiler, M., Waldherr, K., et al. (2018). Web-based indicated prevention of common mental disorders in university students in four European countries - study protocol for a randomized controlled trial. Internet Interventions, 16, 35-42. https://doi.org/10.1016/j. invent.2018.02.004.

Myers, D. G. (2000). The funds, friends, and faith of happy people. American Psychologist, 55(1), 56-67. 
Nicolaisen, M. \& Thorsen, K.(2016). What are friends for? Friendships and loneliness over the lifespan-from 18 to 79 years. The International Journal of Aging and Human Development, 0(0) 1-33.

Pereira, M.G., Taysi, E., Orcan, F. \& Fincham, F. (2014). Attachment, infidelity, and loneliness in college students involved in a romantic relationship: The role of relationship satisfaction, morbidity, and prayer for the partner. Contemporary Family Therapy, volume 36, pages333-350.

Pervin, M. M., \& Ferdowshi, N. (2016). Suicidal ideation in relation to depression, loneliness, and hopelessness among university students. The Dhaka University Journal of Biological Sciences, 25(1), 57-64.

Pinquart, M. (2003). Loneliness in married, widowed, divorced, and never-married older adults. Journal of Social and Personal Relationships, 20, 31-53.

Rahman, A., Bairagi, A., Kumar Dey, B. \& Nahar, L. (2017). Loneliness and depression in university students. Chittagong University. Journal of Biological Science, vol-7 (1\&2), 2012, 175-189.

Rowsell, H. C., \& Coplan, R. J. (2013). Exploring links between shyness, romantic relationship quality, and well-being. Canadian Journal of Behavioural Science / Revue canadienne des sciences $d u$ comportement, 45(4), 287295. https://doi.org/10.1037/a0029853.

Russell, D. W. (1996). UCLA loneliness scale (Version 3): Reliability, validity, and factor structure. Journal of Personality Assessment, $66 \quad$ (1), 2040. https://doi.org/10.1207/s15327752jpa6601_2.

Sağkal, A. S. ve Özdemir, Y. (2018). Algılanan romantik ilişki kalitesi ölçeği’nin (ARIKÖ) Türkçe'ye uyarlanması: Geçerlik ve güvenirlik çalışması. Mehmet Akif Ersoy Üniversitesi Eğitim Fakültesi Dergisi, 46, 22-40. doi: 10.21764/maeuefd.329888.

Satıc1, S.A.(2019). Facebook addiction and subjective well-being: A study of the mediating role of shyness and loneliness. International Journal of Mental Health and Addiction, volume 17, pages41-55.

Satıcı, S.A., Uysal. D. \& Deniz, M.E.(2016). Linking social connectedness to loneliness: The mediating role of subjective happiness. Personality And Individual Differences, vol.97, pp.306-310, 2016.

Saunders, H., Kraus, A., Barone, L., \& Biringen, Z. (2015). Emotional availability: Theory, research, and intervention. Frontiers in Psychology, 6, Article 1069. 
Seçim, Ö., Alpar, Ö. \& Algür, S. (2014). Üniversite öğrencilerinde yalnızlık: Akdeniz Üniversitesinde yapılan ampirik bir araştırma. Elektronik Sosyal Bilimler Dergisi, Cilt:13 Say1:48, ss.200-215.

Seligman, M., \& Csikszentmihalyi, M. (2000). Positive psychology: An introduction. American Psychologist, 55, 5-14.

Shulman, S., \& Connolly, J. (2013). The challenge of romantic relationships in emerging adulthood: Reconceptualization of the field. Emerging Adulthood, 1(1), 2739. https://doi.org/10.1177/2167696812467330.

Simon, R. W. (2002). Revisiting the relationships among gender, marital status, and mental health. American Journal of Sociology,107, 1065 - 1096.

Simon, R. W., \& Barrett, A. E. (2010). Nonmarital romantic relationships and mental health in early adulthood: Does the association differ for women and men? Journal of Health and Social Behavior, 51(2), 168-182. doi:10.1177/0022146510372343.

Sternberg, R. J. (1986). A triangular theory of love. Psychological Review, 93(2), 119135. https://doi.org/10.1037/0033-295X.93.2.119.

Suldo, S.M., \& Fefer, S. (2015). Parent-child relationships and well-being. In C Proctor \& P.A Linley (Eds). Research applications and interventions for children and adolescents: A positive psychology perspective (pp.131-147). New York. Springer.

Stice, L.V. \& Lavner, J.A.(2018). Social connectedness and loneliness mediate the association between autistic traits and internalizing symptoms among young adults. Journal of Autism and Developmental Disorders, 49, 1096-1110.

Olson, K. L., \& Wong, E. H. (2001). Loneliness in marriage. Family Therapy, 28, 105-112.

O’Rourke, H.M., Collins, L. \& Sidani, S.(2018). Interventions to address social connectedness and loneliness for older adults: A scoping review. BMC Geriatrics, $18: 214$.

Özdemir, U., \& Tuncay, T. (2008). Correlates of loneliness among university students. Child and Adolescent Psychiatry and Mental Health, 2, Article 29. https://doi.org/10.1186/1753-2000-2-29.

Tabachnick, B. G, Fidel, L. S. (2007). Using Mulivariate Statistics. MA: Allyn\&Bacon, Inc. Telef, B. B. (2013). Psikolojik iyi oluş ölçeği: Türkçeye uyarlama, geçerlik ve güvenirlik çalışması [The adaptation of psychological well-being into Turkish: A validity and reliability study]. Hacettepe University Journal of Education, 28(3), 374-384. 
Tooby J., Cosmides L. (1992). "The psychological foundations of culture," in The Adapted Mind: Evolutionary Psychology and the Generation of Culture, eds Barkow J. H., Cosmides L., Tooby J., editors. (Oxford: Oxford University Press; ), 19-136.

Tu, Y. \& Zhang, S. (2014). Loneliness and subjective well-being among chinese undergraduates: The mediating role of self-efficacy. Social Indicators Research, DOI 10.1007/s11205-014-0809-1.

Türk, E.G. \& Yıldız, A.D. (2017). Aşk biçemleri, ilişki doyumu ve yalnızlık: Üniversite öğrencileri üzerine bir çalışma. Türk Psikolojik Danışma ve Rehberlik Dergisi, (48):97-109.

Umberson, D., \& Karas Montez, J. (2010). Social relationships and health: A flashpoint for health policy. Journal of Health and Social Behavior, S54-S66.

Varghese, M.H \& Pistole, M.C. (2017). College student cyberbullying: Self-Esteem, depression, loneliness, and attachment. Journal of College Counseling, 20.

Wang, J., Mann, F., Lloyd-Evans, B., Ma, R. \& Johnson, S. (2018). Associations between loneliness and perceived social support and outcomes of mental health problems: A systematic review. BMC Psychiatry, 18:156.

Weiss, R. S. (1973). Loneliness: The experience of emotional and social isolation. Cambridge, MA: MIT Press.

Yalçın, İ. (2015). İyi oluş ve sosyal destek arasındaki ilişkiler: Türkiye'de yapılmış çalışmaların meta analizi [Relationships between well-being and social support: A meta analysis of studies conducted in Turkey]. Türk Psikiyatri Dergisi, 26(1), 21-32.

Zhao, J., Kong, F. \& Wang, Y.(2013). The role of social support and self-esteem in the relationship between shyness and loneliness. Personality and Individual Differences, Volume 54, Issue 5, April 2013, Pages 577-581.

Zimmer-Gembeck, M. J. \& Petherick, J. (2006). Intimacy, dating goals, and relationship satisfaction during adolescence and emerging adulthood: Identity formation, age, and sex as moderators. International Journal of Behavioral Development, 30(2), 167177. doi: 10.1177/0165025406063636. 